\title{
Soft and Hard X-ray SASE Free Electron Lasers
}

\author{
Siegfried Schreiber \\ Deutsches Elektronen-Synchrotron, \\ Notkestr. 85, 22603 Hamburg, Germany \\ siegfried.schreiber@desy.de
}

\begin{abstract}
In the last couple of years, free electron lasers (FELs) have been a remarkable success as fourth generation light sources all over the world. Operating in the SASE mode, they produce laser-like radiation in a broad wavelength range. Especially in the soft and hard X-ray ranges, these light sources open unique and completely new fields in physics and allow a vast range of applications in most scientific fields. This article gives an overview of the principles of FELs and the SASE process, discusses technological challenges and solutions, and presents an outlook for future developments.
\end{abstract}

Keywords: Free electron lasers; high-gain FEL process; SASE; accelerators; FLASH.

\section{Introduction}

Free electron lasers (FELs) were proposed by Madey in 1971 [1]. It took until 1976 for the first observation of stimulated emission of radiation by relativistic electrons to be reported [2]. The experiment was conducted at the W. W. Hansen High Energy Physics Laboratory (HEPL) using an electron beam from the Stanford University superconducting accelerator. The radiation with a wavelength of $10.6 \mu \mathrm{m}$ was produced in a helical undulator.

In 1977, the same group succeeded for the first time in operating an FEL oscillator [3]. A wavelength of $3.4 \mu \mathrm{m}$ was achieved with an average power of $0.36 \mathrm{~W}$.

Since then, many other facilities have been constructed and operated as storage-ring- or linac-based FELs, from the $\mathrm{THz}$ frequency range and micrometer wavelength range down to the visible and nearUV. Some of them were built as energy recovery linacs.

With the establishment of the principles of selfamplified spontaneous emission (SASE), where the amplification process starts from spontaneous radiation in a long undulator, the path to wavelengths in VUV, soft, and hard X-rays was opened. The SASE process was first described by Saldin and Kondratenko [4], and theoretically explored in the early 1980 s by many groups $[5-11]$.
Recent advances in high-brightness electron sources, precise magnetic undulator devices, and bunch compression techniques have made it possible to construct and experimentally confirm operation of X-ray FELs based on the SASE principles.

These facilities provide femtosecond short FEL pulses with a peak brilliance exceeding $10^{30}$ photons/ ( $\mathrm{s} \mathrm{mad}^{2} \mathrm{~mm}^{2} 0.1 \%$ bw) (Fig. 1).

The scientific potential of X-ray lasers is overwhelming and led to the realization of major large facilities all over the world: LCLS, located at SLAC, USA [12] (in operation); XFEL, located at SPring-8, Japan [13]; and the European XFEL, located at DESY, Germany [14] (both under construction). Pilot facilities were constructed and operated with great success: FLASH at DESY [15] and SCSS at SPring-8 [13]. Many other projects aiming for X-rays are in the planning phase or have been proposed, like FERMI at Elettra, Italy [16]; the SwissFEL at PSI, Switzerland [17]; or the New Light Source (NLS) proposal in the UK [18].

A list of FELs worldwide can be found in [19]. It includes FELs in the long wavelength range ( $\mu \mathrm{m}$ to $\mathrm{mm})$, which are not discussed here in detail.

In 1985, a high-gain free electron amplifier with high extraction efficiency was operated at the Electron Laser Facility (ELF) at the Lawrence Livermore Laboratory [20]. Exponential gain and 


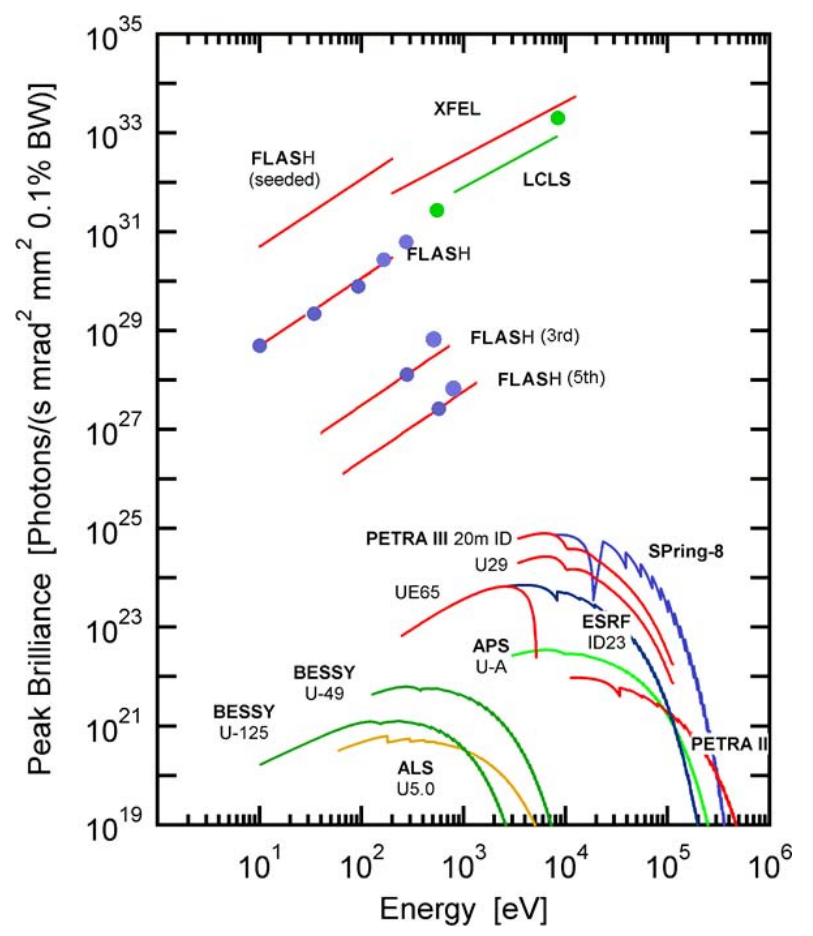

Fig. 1. Peak brilliance of soft and hard X-ray FELs compared to some third generation light sources. Examples of experimental data of FLASH (blue dots) and LCLS (green dots) are included. Lines are estimates from calculations.

saturation was achieved at a wavelength in the microwave range with a frequency of $34.6 \mathrm{GHz}$.

In 1997, the SASE amplification process was for the first time demonstrated at a wavelength of $16 \mu \mathrm{m}$ at the UCLA Saturnus linac [21], and at $15 \mu \mathrm{m}$ at the Advanced Free-Electron Laser (AFEL) linac of the Los Alamos National Laboratory [22]. Later on, using the UCLA-Kurchatov undulator from the Saturnus experiment, a gain larger than $10^{5}$ at a wavelength of $12 \mu \mathrm{m}$ was achieved at AFEL [23].

In September 2000, saturation in the visible $(530 \mathrm{~nm})$ and the UV $(390 \mathrm{~nm})$ of an SASE FEL was demonstrated at the low energy undulator test line LEUTL at the Argonne National Laboratory (ANL) [24].

Many experiments followed in the visible and the near-UV range; see for instance [25-27].

In February 2000, a group at the TESLA Test Facility (TTF) at DESY demonstrated lasing at $109 \mathrm{~nm}$, and in September 2001 saturation in the VUV at $98 \mathrm{~nm}$ with the TTF1 FEL [28-30].

In 2003 and 2004, the TTF1 FEL was substantially upgraded to the FEL user facility FLASH.
Since August 2005, FLASH has operated as a user facility and provided coherent, femtosecond short laser pulses in the EUV and soft X-ray wavelength range between 47 and $6.9 \mathrm{~nm}$ (fundamental) [31-33]. Recently, it has been upgraded to a beam energy of $1.2 \mathrm{GeV}$ and has demonstrated lasing at a wavelength of $4.45 \mathrm{~nm}$ [34].

The facility is hosting many international groups exploiting the unique aspects of this source in projects ranging from atomic physics through materials science to biology [35].

In June 2006, the SCSS prototype accelerator for the XFEL at SPring- 8 achieved its first lasing using the SASE process at a wavelength of $49 \mathrm{~nm}$ [36], and saturation between 50 and $60 \mathrm{~nm}$ shortly after [37]. Since October 2007, this facility has also served user experiments.

The first SASE FEL producing hard X-rays is the Linac Coherent Light Source (LSLC) at SLAC. In April 2009, lasing and saturation at a wavelength of $0.15 \mathrm{~nm}$ was demonstrated [38]. Since September 2009, LCLS has been run as a user facility providing the worldwide shortest FEL wavelength with about $2 \cdot 10^{12}$ photons per pulse and pulse duration in the range of $70-100 \mathrm{fs}$ (FWHM) [39].

In order to make these facilities a reality, major technological challenges and theoretical problems needed to be addressed and solved. Most important was the required excellent quality of the electron beam in terms of transverse emittance, peak current, and energy spread. New electron sources needed to be developed in order to achieve the required normalized transverse emittance below $1 \mu \mathrm{m}$ for $0.1-1 \mathrm{nC}$ bunch charge. For this, most facilities have chosen the RF-gun-based photoinjector. As an example, FLASH and LCLS are using L-band and S-band $\mathrm{RF}$ guns respectively, reaching the emittance goal of $\approx 1 \mu \mathrm{m}$ (normalized) [40, 41].

To obtain high peak currents in the order of several kA, novel bunch compression schemes have been developed. It turned out that space charge and bunch instabilities due to coherent radiation strongly affect the beam quality during and after compression. After many theoretical studies and simulations, we now start to understand the complicated dynamics of $\mathrm{kA}$ type beams.

In order to experimentally measure these effects, in both the transverse and the longitudinal phase space, new experimental methods have 
been developed: sophisticated techniques to measure small transverse emittance at the electron source, and methods to measure bunch duration and longitudinal shape in the $10 \mathrm{fs}$ range.

In some cases, major developments of the drive laser have been made: transverse and especially longitudinal shaping of the laser pulses. For the burst mode FELs, high average power lasers together with high efficiency cathodes are required. New types of photocathodes with high quantum efficiency together with a good lifetime and robustness needed to be developed.

One facility showed, that also pulsed injectors based on heated cathodes with thermionic electron emission together with an appropriate bunching scheme can provide sufficient beam quality [42].

In the field of diagnostics of the FEL radiation, many new developments have been made: for example, the measurement of FEL radiation over several orders of magnitude from the spontaneous radiation to saturation. To monitor radiation properties during experiments, absolutely calibrated devices to nondestructively measure single pulse radiation energies and wavelength spectra are a must nowadays.

Many articles and books have been published on FELs. This article will follow the excellent introduction to UV and soft X-ray FELs by Schmüser, Dohlus, and Rossbach [43]. A comprehensive overview of the physics of FELs can be found in [44], or in articles in Vol. 6 of Laser Handbook $[45,46]$. I would also like to draw the reader's attention to the recently published articles by Huang and Kim [47] and by Barletta et al. [48] giving an overview of recent developments and ideas on hard X-ray FELs and novel seeding schemes.

\section{The SASE Process}

The SASE process introduces a high-gain amplification starting from spontaneous undulator radiation. In a long undulator system, the spontaneous radiation acts as a seed initiating the amplification process. An equivalent approach especially useful for calculations is to start with a random electron distribution in the electron bunch. The current density then contains a noise term which has a spectral component within the FEL bandwidth. The narrow-band spectral component acts as a seed for the amplification process. The amplified radiation features full transverse coherence with a brightness several orders of magnitude higher than spontaneous radiation. The high brightness is mainly due to high gain in the amplification process and due to the ultrashort pulses generated, which are in the 10-100 fs range.

Besides the high brilliance and femtosecond short pulses, another striking property of SASE FELs is the tunability in wavelength down to hard X-rays. With the SASE amplification process, the generation of laser-like coherent radiation in the angstrom wavelength range has been made possible.

FELs in the low-gain regime use oscillator schemes to amplify the radiation. The oscillator can be integrated into a storage ring. An example is the FEL Elettra at the Sincrotrone Trieste. Elettra is operating at wavelengths in the UV down to $190 \mathrm{~nm}$ [49], where mirrors to form the resonating oscillator cavity are readily available.

Due to the lack of suitable mirrors in a wide range of wavelengths, especially in the VUV, soft and hard X-ray regime, the oscillator scheme is not applicable. Recently, possibilities of using Bragg reflectors in an oscillator design for hard X-rays have been under discussion [50, 51].

In the following, starting with an overview of undulator radiation and high-gain amplification, the SASE principle is described and electron beam properties required to successfully operate an SASE FEL are derived.

\section{Undulator Radiation}

Electrons emit radiation in bending magnets of accelerators. In high-energy synchrotrons or storage rings, this radiation also covers VUV wavelength, soft and hard X-rays, and is therefore attractive for photon science experiments.

\subsection{Radiation from a bunch of electrons}

The relativistic electrons are accelerated toward the center of the bend and emit synchrotron radiation tangentially to the orbit. Normally all electrons emit independently of each other, leading to incoherent radiation with a continuous frequency spectrum from zero to a little beyond the critical frequency $\nu_{\mathrm{c}}$;

$$
\nu_{\mathrm{c}}=\frac{3 c \gamma^{3}}{4 \pi r},
$$


where $r$ is the radius of curvature of the bending magnet, and $\gamma$ is the Lorentz factor:

$$
\gamma=\frac{W}{m_{\mathrm{e}} c^{2}} .
$$

$W$ is the energy of the electron. ${ }^{\text {a }}$ The radiated power of a single electron is

$$
P_{\mathrm{sync}}=\frac{e^{2} c \gamma^{4}}{6 \pi \epsilon_{\mathrm{o}} r^{2}}
$$

As usual, $c$ is the speed of light in vacuum, $e$ the electron charge magnitude, and $\epsilon_{\mathrm{o}}$ the permittivity of free space; the convention of SI units is used.

In a bunch of $N_{\mathrm{e}}$ electrons, where all electrons radiate independently, the radiated power is proportional to the square of the electron charge times the number of electrons in that bunch:

$$
P_{\text {incoh }}=N_{\mathrm{e}} P_{\text {sync }} \propto N_{\mathrm{e}} e^{2} .
$$

The electrons in a bunch start to emit coherently for wavelengths $\lambda_{\mathrm{s}}$ comparable to or larger than the bunch length $\sigma_{z}$. We assume that the transverse bunch size is sufficiently small as well.

For $\sigma_{z} \gg \lambda_{\mathrm{s}}$, the bunch containing $N_{\mathrm{e}}$ electrons acts like a single macroparticle with a charge of $Q_{b}=-N_{\mathrm{e}} e$. Since the radiated power is proportional to the square of the particle charge [Eq. (3)], the radiation is now emitted coherently with a power proportional to $Q_{b}^{2}=\left(N_{\mathrm{e}} e\right)^{2}=N_{\mathrm{e}}\left(N_{\mathrm{e}} e^{2}\right)$, surpassing the incoherent radiation of $N_{\mathrm{e}}$ electrons by a factor of $N_{\mathrm{e}}$ :

$$
P_{\text {coh }}=N_{\mathrm{e}} P_{\text {incoh }} .
$$

With $N_{\mathrm{e}}$ being typically on the order of $10^{6}$, the increase in radiation power can be remarkably large.

In practice, typical electron bunch lengths are on the order of millimeters or centimeters. The frequency of coherent synchrotron radiation is usually in the $\mathrm{THz}$ range. Since the spectrum of coherent radiation is a function of the bunch length, it is used in many facilities to estimate the longitudinal bunch length or shape.

It turns out that the coherent part of the synchrotron radiation is important for SASE FELs in two aspects. Firstly, it is the base of the SASE principle itself. As we will see later, the interaction of the electron bunch with the undulator field leads to the formation of microbunches which emit coherent intense radiation. Secondly, the dynamics of the electron bunch during the compression phase is strongly affected by the emission of coherent synchrotron radiation in the magnetic chicane bends.

\subsection{Radiation from undulators}

Modern synchrotron radiation sources use undulators to significantly improve the brilliance, compared to simple synchrotron radiation from dipoles. A periodic series of small bending magnets of alternating polarity with a period $\lambda_{\mathrm{u}}$, the undulator period, are placed along the electron beam path $z$ such that $B(z)=B_{\mathrm{o}} \cos \left(2 \pi z / \lambda_{\mathrm{u}}\right) . B_{\mathrm{o}}$ is the peak magnetic field on the undulator axis. As illustrated in Fig. 2, forced by the oscillating magnetic field, the electrons move on a sinusoidal path, and the net deflection vanishes. The amplitude of the oscillating path is small, so that the light is allowed to interfere along the undulator. The beauty of the undulator radiation is that it not only appears in a small cone of $\approx 1 / \gamma$, but also consists of narrow spectral lines with high brilliance.

The wavelength of emitted undulator radiation is usually derived using the Lorentz contraction of the undulator period, considering the relativistic Doppler shift, and taking into account details of the sinusoidal trajectory [43]. The relativistic length contraction reduces the undulator period to $\lambda_{\mathrm{e}}=\lambda_{\mathrm{u}} / \gamma$.

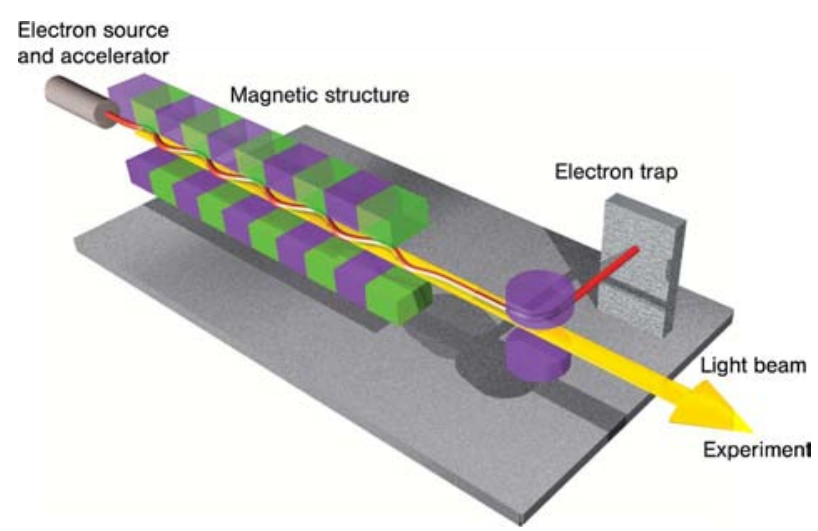

Fig. 2. Sketch of an electron beam passing through an undulator. Shown is a setup typical for an SASE FEL. In storage rings, the undulator would be placed into the circulating electron beam. The sinelike trajectory is exaggerated for clarity. (Source: DESY.) 
The electrons moving along the planar undulator oscillate with the frequency $\omega_{\mathrm{e}}=2 \pi c \gamma / \lambda_{\mathrm{u}}$ and emit radiation like an oscillating dipole. For an observer looking against the electron beam, the relativistic Doppler effect shifts the observed radiation to $\lambda_{\mathrm{s}}=$ $\lambda_{\mathrm{e}} / 2 \gamma=\lambda_{\mathrm{u}} / 2 \gamma^{2}$. The effects of the sinusoidal trajectory are taken into account by introducing the dimensionless undulator parameter $K$ :

$$
K=\frac{e B_{\mathrm{o}} \lambda_{\mathrm{u}}}{2 \pi m_{\mathrm{e}} c} .
$$

$B_{\mathrm{O}}$ is the peak magnetic field on the undulator axis. Helical undulators have a slightly modified $K$ parameter: $K_{\text {hel }}=\sqrt{2} K$.

This leads to the important formula for the fundamental wavelength of the radiation of a planar undulator in the forward direction

$$
\lambda_{\mathrm{s}}=\frac{\lambda_{\mathrm{u}}}{2 \gamma^{2}}\left(1+\frac{K^{2}}{2}\right) .
$$

The electrons are slightly retarded with respect to the light wave; however, the emitted radiation with the wavelength $\lambda_{\mathrm{s}}$ is just phase-matched with the oscillation of the electrons in the undulator field.

Phase matching holds also for higher odd harmonics:

$$
\lambda_{n}=\frac{\lambda_{\mathrm{s}}}{n}, \quad n=1,3,5, \ldots
$$

Narrow spikes in the spectral distributions are formed. In the forward direction, even harmonics are suppressed by destructive interference.

An important consequence of Eq. (7) is the apparently unlimited tunability of the radiation by simply choosing the electron beam energy $W=$ $\gamma m_{\mathrm{e}} c^{2}$.

To a certain extent, tuning is also possible by changing the magnetic field strength $B_{\mathrm{o}}$. Many wigglers or undulators for long wavelengths, where tolerances are not too tight, use electromagnets. Here the field strength is simply varied with the coil current. Undulators with tight tolerances, high magnetic fields, and short periods are usually built using permanent magnets. Here the field strength can be varied by opening or closing the gap of the undulator magnet arrangement. As the name suggests, fixed gap undulators with permanent magnets like the FLASH undulator cannot change their field strength. Laterally tapered undulators, like the LCLS undulator, have the possibility of tuning the $K$ value by laterally moving the undulator without the need to install a technically demanding variable gap.

To give an example, the wavelength of the undulator radiation of an undulator with a period of $25 \mathrm{~mm}$ and an undulator parameter of $K=1$ $\left(B_{\mathrm{o}}=0.43 \mathrm{~T}\right)$ can be varied from $5 \mathrm{~nm}$ for a beam energy of $1 \mathrm{GeV}$ to $0.14 \mathrm{~nm}$ for $6 \mathrm{GeV}$. This, however, also shows that with state-of-the-art undulators, electron beam energies well above $1 \mathrm{GeV}$ are required to obtain radiation in the soft-to-hard $\mathrm{X}$-ray regime.

As pointed out in [43], it can be shown that the wavelength of the undulator radiation $\lambda_{\mathrm{s}}$ in the forward direction is equal to the light wavelength $\lambda_{\mathrm{r}}$, yielding constant energy transfer between the bunch and the amplified light. This is the reason why the amplification process in an FEL can be seeded by the spontaneous radiation itself. This is the basis of the SASE process.

\section{The High-Gain FEL Process}

The high-gain FEL process introduces a new feature compared to low-gain amplification: the electron bunch itself is bunched in a length scale of the wavelength of the undulator radiation, making it possible to emit coherent radiation orders of magnitude more intense than spontaneous radiation. The amplification process eventually leads to saturation in a single pass through the undulator, which is also an important phenomenon inherent in the high-gain FEL process.

In the following, we will briefly discuss the highgain FEL process, and, in the next section, the SASE process. We then derive important requirements for the accelerator, the undulator system, and especially on the electron bunch itself.

\subsection{Coherent emission and microbunching}

As discussed in the previous section, coherent emission of radiation requires an electron bunch length smaller than the wavelength $\lambda_{\mathrm{s}}$ of the radiation. Producing a bunch with $N_{\mathrm{e}}=10^{9}$ electrons with a length of for example $10 \mathrm{~nm}$ is obviously not feasible. Space charge forces would quickly disrupt the bunch.

Only the process of microbunching during the passage of the electron bunch through the undulator field makes it possible to achieve nanometer-scale 
density modulation within the bunch itself. Electrons transferring energy to the light wave will lose momentum and thus travel a longer sinusoidal path along the undulator, since

$$
p=e B_{\mathrm{o}} r,
$$

with the electron momentum $p ; r$ is the radius of curvature of the circular path in the magnetic undulator field $B_{\mathrm{o}}$. Due to the smaller radius of curvature, the amplitude of the sinelike oscillation is larger. As a consequence, these electrons fall behind those electrons which gain energy from the light wave.

While the bunch is traveling along the undulator, an initial weak microbunching leads to an increase in emitted radiation. More and more energy is now transferred to the light wave, which results in a growing modulation. This process is self-sustained and leads to exponentially growing power of the emitted light wave.

The process eventually leads to a concentration of electrons in small longitudinal slices smaller than the radiated wavelength. The distance of the slices is just the undulator wavelength $\lambda_{\mathrm{s}}$ from Eq. (7). This is illustrated by a simulation of the microbunching mechanism in Fig. 3. With full modulation, the process eventually reaches saturation.

Because all electrons in a microbunch radiate coherently and many microbunches radiate coherently with respect to each other, the increase in radiation power is with Eq. (5) on the order of the total number of electrons in all microbunches within the coherence length. The number of electrons $N_{\mathrm{e}}$ can be a large number, which explains the enormously high single pass gain on the order of $10^{6}$ or more. Figure 4 shows as an example the measured radiation energy at the SASE FEL of the TESLA Test Facility along the undulator at a wavelength of $98 \mathrm{~nm}$ [30]. The energy in the radiation pulse starts from spontaneous emission in the $\mathrm{nJ}$ level to almost $100 \mu \mathrm{J}$ at saturation. This is a total gain of $10^{5}$.

In the following, we give an overview of the onedimensional FEL theory and discuss the relevant parameters and results. A more complete overview of the $1 \mathrm{D}$ theory can be found in many reviews, for instance [43].

\subsection{One-dimensional FEL theory}

As explained in [43], the one-dimensional FEL theory starts from the coupled pendulum equations. These describe the phase space motion of the electrons in the electric field of the light wave. A self-consistent treatment of the high-gain FEL process includes also the inhomogeneous wave equation for the electric field of the light wave, and describes the evolution of the density modulation along the bunch taking longitudinal space charge forces into account.

The 1D theory neglects the dependences of the bunch charge density and the electromagnetic fields on the transverse coordinates. The electron bunch is assumed to have a homogeneous charge distribution with a sufficiently large radius. The bunch is assumed

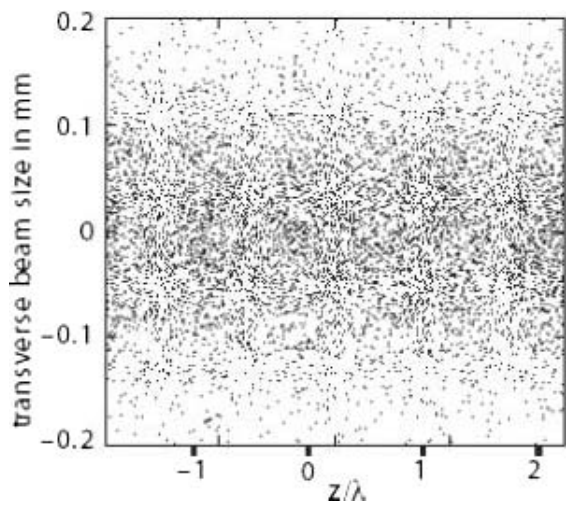

(a)

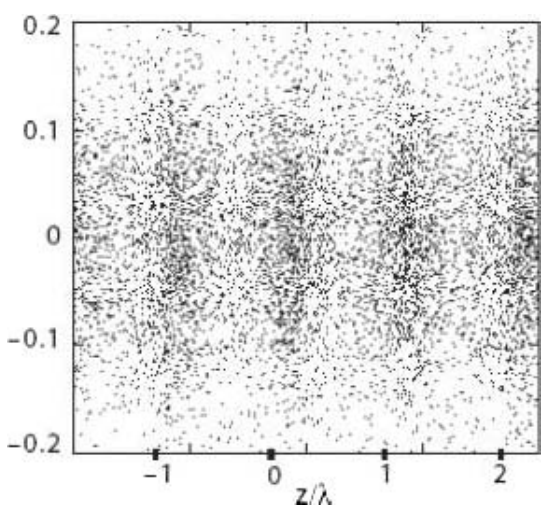

(b)

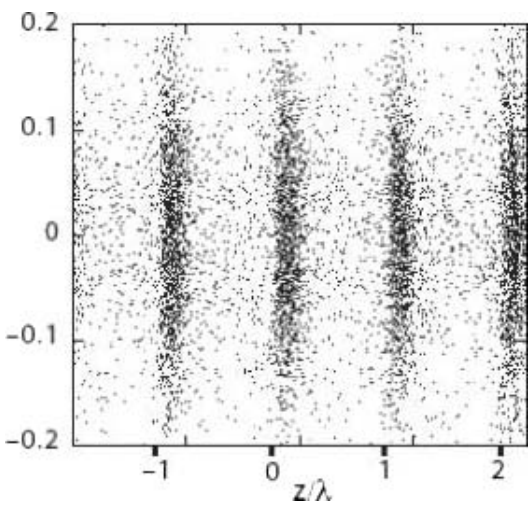

(c)

Fig. 3. Numerical simulation of microbunching. Particles within the bunch are plotted with respect to their transverse and longitudinal coordinates in the bunch; the latter is normalized to the wavelength $\lambda=\lambda_{\mathrm{s}}$ of the emitted radiation. The microbunching develops when traveling along the undulator. The initially uniform distribution (a) develops a microbunching (b), which is fully developed at the end of the undulator (c). The distance of the microbunches is equal to the emitted light wavelength $\lambda_{\mathrm{s}}$. (Courtesy of Sven Reiche, PSI.) 


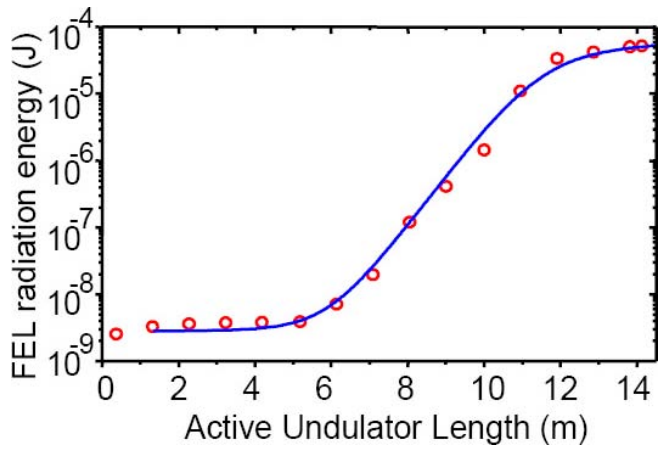

Fig. 4. Exponential growth of the SASE FEL radiation along the undulator. The experimental data (dots) are measured with the TTF FEL at a wavelength of $98 \mathrm{~nm}$. The solid line is from a simulation of the amplification process. The power gain length is $L_{\mathrm{g} 0}=67 \pm 5 \mathrm{~cm}$, the FEL pulse energy at saturation $90 \mu \mathrm{J}$. (Adapted from Refs. 30 and 95.)

to be very long, so that effects occurring at the head or tail can be neglected. The peak current $I_{0}$ of a bunch with a homogeneous longitudinal charge distribution of $N_{\mathrm{e}}$ electrons and a duration of $T$ is thus simply given by

$$
I_{0}=\frac{e N_{\mathrm{e}}}{T} .
$$

The $1 \mathrm{D}$ theory is not sufficient to describe the full physics of VUV and X-ray SASE FELs. However, it has analytic solutions and allows one to derive some important parameters so as to understand the principles of the SASE FEL process. Simulations using a full 3D model and its consequences for the bunch parameter tolerances will be discussed later.

\subsubsection{Third-order equation}

The 1D model leads to a set of coupled first-order equations which describe the time evolution of the ponderomotive phases and energy deviations of the electrons, as well as the time evolution of the current density and the amplitude of the radiated light wave. We will not discuss these equations here and will refer to the literature. An extensive discussion can be found for example in [43]. This set of equations describes the basic features of the high-gain FEL process, including exponential growth of radiated power and saturation.

The coupled first-order equations can only be solved numerically. Assuming a small modulation of the current density, a linear third-order differential equation for the electric field can be derived. This equation has the advantage that it can be solved analytically and that it introduces important parameters useful for understanding the basic physics of the high-gain FEL process.

The third-order differential equation contains the complex electric field amplitude $E(z)$ as a function along the path length $z$ :

$$
\frac{E^{\prime \prime \prime}}{\Gamma^{3}}+2 i \frac{\eta}{\rho_{\mathrm{fel}}} \frac{E^{\prime \prime}}{\Gamma^{2}}+\left(\frac{k_{p}^{2}}{\Gamma^{2}}-\left(\frac{\eta}{\rho_{\mathrm{fel}}}\right)^{2}\right) \frac{E^{\prime}}{\Gamma}-i E=0 .
$$

The prime' stands for the derivative of the complex electric field $E$ with respect to the longitudinal coordinate $z\left(E^{\prime}=d E / d z\right)$,

$$
\eta=\frac{W-W_{\mathrm{r}}}{W_{\mathrm{r}}}
$$

which is the relative energy deviation of the beam from the resonance energy $W_{\mathrm{r}}$.

Let us consider the case of seeding the FEL process with a seed field with a wavelength $\lambda_{\mathrm{s}}=\lambda_{\mathrm{r}}$. The resonance energy $W_{\mathrm{r}}=\gamma_{\mathrm{r}} m_{\mathrm{e}} c^{2}$ is then derived from the undulator equation (7):

$$
\lambda_{\mathrm{r}}=\frac{\lambda_{\mathrm{u}}}{2 \gamma_{\mathrm{r}}^{2}}\left(1+\frac{K^{2}}{2}\right) .
$$

An electron beam with the energy $W_{\mathrm{r}}$ will just radiate with the wavelength $\lambda_{\mathrm{r}}$, which is equal to the seed wavelength. The energy of the electrons may deviate a little from the resonance energy $W_{\mathrm{r}}$, which is accounted for by the energy deviation or detuning parameter $\eta$ defined in Eq. (12).

The third-order equation contains four important parameters: the gain parameter $\Gamma$, the space charge parameter $k_{p}$ (both with a dimension of an inverse length), the dimensionless FEL or Pierce parameter $\rho_{\mathrm{fel}}$, and the power gain length $L_{\mathrm{g} 0}$ :

$$
\begin{aligned}
\Gamma & =\left(\frac{\pi \mu_{\mathrm{o}} K_{\mathrm{JJ}}^{2} e^{2} n_{e}}{2 \lambda_{\mathrm{u}} \gamma_{\mathrm{r}}^{3} m_{\mathrm{e}}}\right)^{1 / 3}, \\
k_{p} & =\sqrt{\frac{2 \lambda_{\mathrm{r}}}{\lambda_{\mathrm{u}}}} \cdot \frac{\omega_{p}}{c}, \\
\rho_{\mathrm{fel}} & =\Gamma \frac{\lambda_{\mathrm{u}}}{4 \pi}=\frac{1}{4 \pi \sqrt{3}} \cdot \frac{\lambda_{\mathrm{u}}}{L_{\mathrm{g} 0}} \\
L_{\mathrm{g} 0} & =\frac{1}{\sqrt{3} \Gamma}=\frac{1}{4 \pi \sqrt{3}} \cdot \frac{\lambda_{\mathrm{u}}}{\rho_{\mathrm{fel}}},
\end{aligned}
$$

with the permeability of free space $\mu_{\mathrm{o}}$ (SI units).

The electron density $n_{e}$ is defined as the number of electrons per unit volume. 
The parameter $K_{\mathrm{JJ}}$ in Eq. (14) is the modified undulator parameter taking the oscillatory coupling between the beam and the light wave into account. For a planar undulator one obtains

$$
K_{\mathrm{JJ}}=K \cdot\left(J_{0}(Y)-J_{1}(Y)\right) .
$$

$J_{0}$ and $J_{1}$ are Bessel functions of the first kind with the argument $Y=K^{2} /\left(4+2 K^{2}\right)$. The correction is small $\left(J_{0} \approx 1\right.$ and $\left.J_{1} \approx 0\right)$; for instance, for $K=1$ the modified $K$ is $K_{\mathrm{JJ}}=0.91$. For helical undulators, $K_{\mathrm{JJ}}=K$.

The gain parameter $\Gamma$ and the space charge parameter $k_{p}$ depend on the beam properties and the layout of the undulator.

The space charge parameter is expressed with the plasma frequency $\omega_{p}$ in the relativistic electron bunch:

$$
\omega_{p}=\sqrt{\frac{n_{e} e^{2}}{\gamma_{\mathrm{r}} \epsilon_{\mathrm{o}} m_{\mathrm{e}}}} .
$$

As an example, we take the FEL FLASH operating at $1 \mathrm{GeV}$ and a wavelength of $6.5 \mathrm{~nm}$. Typical electron beam parameters are a total bunch length of $80 \mathrm{fs}$, a bunch size of $80 \mu \mathrm{m}$ along the undulator with a charge of $0.2 \mathrm{nC}$ leading to a peak current of $2.5 \mathrm{kA}$. For the undulator we take $K=1.23$ with a period of $\lambda_{\mathrm{u}}=27.3 \mathrm{~mm}$. From the definitions above we compute a gain parameter of $\Gamma=0.75 \mathrm{~m}^{-1}$ and a space charge parameter of $k_{p}=0.1 \mathrm{~m}^{-1}$. The FEL parameter is $\rho_{\mathrm{fel}}=1.6 \cdot 10^{-3}$, and the power gain length $L_{\mathrm{g} 0}=0.77 \mathrm{~m}$.

This example already indicates two main requirements on the electron bunch in order to achieve a suitable amplification gain: high peak current in the $\mathrm{kA}$ range, and small beam sizes along the undulator $(\leq 100 \mu \mathrm{m})$ translating into a small transverse beam emittance. Details will be discussed in the next section.

\subsubsection{Solutions to the third-order equation}

For present-day linac-driven FELs, the space charge parameter $k_{p}$ can be neglected compared to $\Gamma$ in Eq. (11). We consider the special case of $\eta=0$. Let the FEL process be initiated by a monoenergetic incident light wave with the amplitude $E_{\text {in }}$ and the wavelength $\lambda_{\mathrm{s}}$, which defines the resonance wavelength $\lambda_{\mathrm{r}}$. In this case, the solution to Eq. (11) has the form

$$
E(z)=A_{1} e^{\frac{i+\sqrt{3}}{2} \Gamma z}+A_{2} e^{\frac{i-\sqrt{3}}{2} \Gamma z}+A_{3} e^{-i \Gamma z},
$$

with the coefficients $A_{1}=A_{2}=A_{3}=E_{\text {in }} / 3$.

The two last terms exhibit an oscillation and a damped oscillation, respectively. The first term, however, leads to exponentially rising radiation power along the undulator, with the power gain length in the exponent:

$$
P(z) \propto \exp (\sqrt{3} \Gamma z)=\exp \left(\frac{z}{L_{\mathrm{g} 0}}\right) .
$$

As shown with the blue dotted line in Fig. 5, the exponential power rise only starts after a few gain lengths $L_{\mathrm{g} 0}$. The radiation power stays almost constant in the so-called lethargy regime. Initially, the exponential growth competes with the damped oscillation, but it quickly dominates.

It is also possible to show that the amplification process according to the third-order equation can be initiated by a density modulation in the electron bunch rather than a seeding light wave.

Also in this case, after 2-3 gain lengths, the power rise approaches the exponential as given in

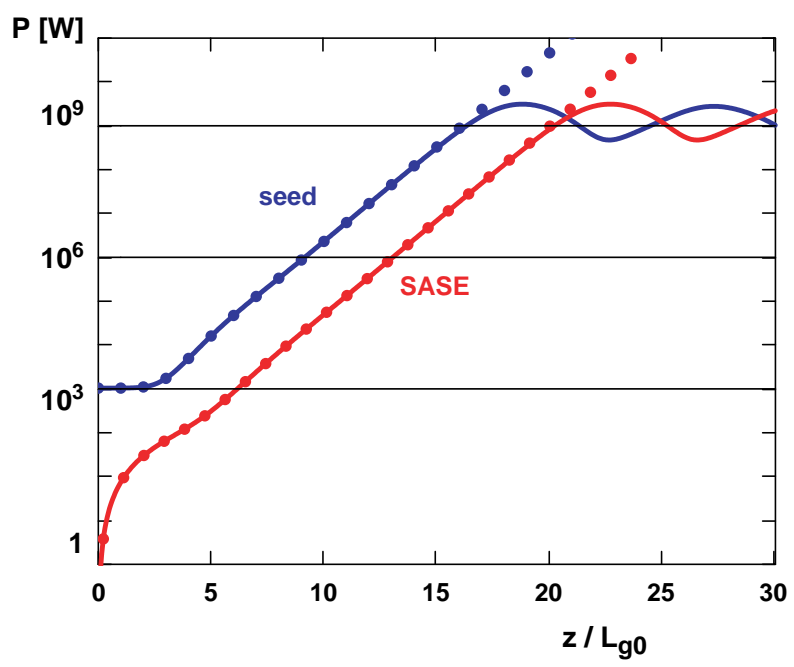

Fig. 5. Exponential growth of the FEL radiation power along the undulator. The curves show the result of a numerical integration of the coupled first-order equations, using a plane wave seed electric field (blue) and initial current density modulation due to random distributions of the electrons in the bunch (SASE FEL, red). After a few gain lengths, the exponential growth starts. In the case of SASE, saturation occurs at $\approx 20 L_{\mathrm{g} 0}$; the seed reduces the saturation length. The saturation power in both cases is equal. The dots represent the analytical solution (20) of the third-order equation (11). (Courtesy of Peter Schmüser, DESY.) 
Eq. (21). Figure 5 shows a calculation of the exponential gain started by a current density modulation in the bunch (red line). For comparison, it also shows the amplification initiated by a seeding field.

Considering the case of different frequencies $\omega=$ $2 \pi c / \lambda$ seeding the amplification process around the resonance $\omega_{\mathrm{r}}$, one can understand the finite bandwidth of the FEL radiation. In this case, the relative energy deviation $\eta$ is small but nonvanishing and is expressed in terms of the seeding frequencies:

$$
\eta(\omega)=-\frac{\omega-\omega_{\mathrm{r}}}{2 \omega_{\mathrm{r}}} .
$$

The gain curve $g(z, \eta)$ is approximated by a Gaussian with an exponentially growing amplitude $\propto$ $\exp \left(z / L_{\mathrm{g} 0}\right)$ and a $z$-depending width in the form $\exp \left(-\eta^{2} / 2 \sigma_{\eta}^{2}\right)$, with $\sigma_{\eta}^{2}=9 \rho_{\mathrm{fel}} L_{\mathrm{g} 0} / 2 z$ :

$$
g(z, \eta) \propto \exp \left(\frac{z}{L_{\mathrm{g} 0}}\right) \cdot \exp \left(\frac{-\eta^{2} z}{9 \rho_{\mathrm{fel}}^{2} L_{\mathrm{g} 0}}\right) .
$$

With Eq. (22) we obtain the rms frequency bandwidth of an SASE FEL [43] $\sigma_{\omega}=2 \omega \sigma_{\eta}$ :

$$
\frac{\sigma_{\omega}(z)}{\omega}=3 \sqrt{2} \rho_{\mathrm{fel}} \sqrt{\frac{L_{\mathrm{g} 0}}{z}}
$$

This expression is valid during the exponential growth of the amplified radiation. From this equation, we obtain a bandwidth after 20 gain lengths of $\sigma_{\omega} / \omega \approx 1.5 \cdot 10^{-3}$ for a typical FEL parameter of $1.6 \cdot 10^{-3}$. The high-gain FEL acts as a narrow-band amplifier.

As we will see later, saturation in the case of SASE is reached after about 20 power gain lengths. We can then approximate the frequency bandwidth at the beginning of saturation with

$$
\frac{\sigma_{\omega}}{\omega} \approx \rho_{\mathrm{fel}} \quad \text { for } \quad z \approx 20 L_{\mathrm{g} 0} .
$$

In contrast to the coupled first-order equations, the third-order equation (11) is not suited for describing a saturation process. However, some form of saturation must occur, since the electrons are losing energy while emitting FEL radiation.

As we will discuss later, it turns out that the FEL efficiency, the ratio of the saturation power $P_{\text {sat }}$ to the beam power $P_{\mathrm{b}}$, is in the order of the FEL parameter $\rho_{\text {fel }}$, independent of the initial seeding power:

$$
\frac{P_{\text {sat }}}{P_{\mathrm{b}}} \approx \rho_{\text {fel }} .
$$

However, in the initial lethargy and the exponential amplification regime, the radiation power depends linearly on the seed power, reaching saturation a couple of gain lengths earlier (large seed) or later (small seed). This is illustrated in Fig. 5, comparing a numerical integration of the coupled firstorder equations for a seeded FEL (large seed) and SASE (small seed).

\section{Self-Amplified Spontaneous Emission}

With the considerations above, it is straightforward to include the startup of the amplification process in a high-gain FEL by the spontaneous radiation itself. In the first section of the undulator, the spontaneous radiation is produced, which later on acts as a seed for the amplification process. This is equivalent to the model, which considers a stochastic density modulation within the electron bunch with a broad white noise spectrum. The appropriate frequency components within the bandwidth of the FEL amplifier [Eq. (24)] are amplified in the remaining section of the undulator.

In the 1D model described above, the third-order equation (11) is now solved with a random current density modulation in the electron bunch taking into account the $z$-depending bandwidth Eq. (24). The FEL power in the exponential gain regime up to saturation is then approximately [43]

$$
P(z)=\frac{m_{\mathrm{e}}^{2} c^{4} \gamma_{\mathrm{r}}^{2}}{3 \sqrt{\pi}} \rho_{\mathrm{fel}}^{2} \omega \sqrt{\frac{L_{\mathrm{g} 0}}{z}} \exp \left(\frac{z}{L_{\mathrm{g} 0}}\right) .
$$

The amplification process is very similar to the case for seeding with an external seed source. Comparing the two, it is possible to estimate the required seed power for external seeding, which needs to be above the self-seed. An expression for the equivalent seeding field $E_{\text {equ }}$ induced by shot noise is given in [43]:

$$
E_{\text {equ }}=\frac{\mu_{\mathrm{o}} c K_{\mathrm{JJ}}}{4 \gamma_{\mathrm{r}} \Gamma} \sqrt{\frac{e I_{0}}{\pi} \sqrt{2 \pi} \sigma_{\omega}} \frac{1}{2 \pi \sigma_{\mathrm{b}}^{2}} .
$$

$I_{\mathrm{o}}$ is the peak current [Eq. (10)] and $\sigma_{\mathrm{b}}$ the rms transverse bunch size.

For the $500 \mathrm{MeV}$ FLASH case $(\lambda=25 \mathrm{~nm})$ and typical parameters, this equivalent seeding field is $0.8 \mathrm{MV} / \mathrm{m}$. In the case of seeding by an external source, this field has to be exceeded, which is a demanding task, especially for shorter wavelengths.

Including the effect of saturation, for instance by using the coupled first-order equations, the solution 
leads to the saturation length $L_{\text {sat }}[47]$ :

$$
L_{\mathrm{sat}} \approx \frac{\lambda_{\mathrm{u}}}{\rho_{\mathrm{fel}}}=4 \pi \sqrt{3} L_{\mathrm{g} 0}=21.8 L_{\mathrm{g} 0} .
$$

This is a very general result which holds with a good approximation within $10 \%$ or $20 \%$ : the saturation length is about 20 power gain lengths. The same approximation has been obtained in [62].

Several experiments have been carried out to measure the gain along the undulator and to demonstrate saturation; refer to the introduction for a brief historical review. Figure 13 shows an example of saturation achieved for a wavelength of $13 \mathrm{~nm}$ at FLASH. The simulation with the code FAST [54] fits well the data. The power gain length obtained from the gain curve is $L_{\mathrm{g}}=1.25 \pm 0.15 \mathrm{~m}$. The $1 \mathrm{D}$ power gain length is calculated to be $L_{\mathrm{g} 0}=0.5 \mathrm{~m}$, suggesting rather large corrections, especially the effect of an energy spread or energy chirp along the bunch. Saturation is achieved after 21 gain lengths, in good agreement with Eq. (29).

Perhaps the most striking example of a gain curve measurement showing saturation is from LCLS. Figure 6 shows the radiation power along the undulator at a wavelength of $1.5 \AA$ [38]. The measured data are overlaid with a simulation by the 3D code GENESIS [53]. The good agreement of the simulation with the data of LCLS (Fig. 6) and

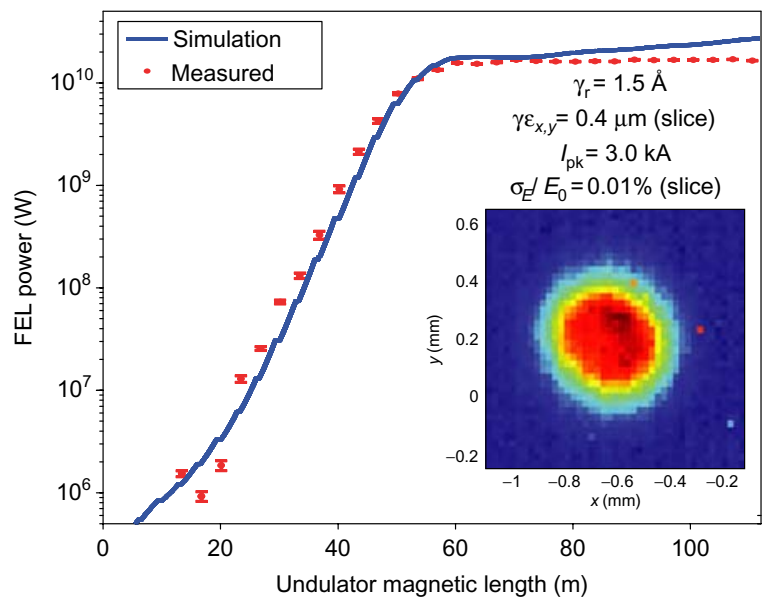

Fig. 6. Radiation power along the undulator at a wavelength of $1.5 \AA$ measured at LCLS (red points). To obtain the gain curve, the amplification is sequentially switched off after each undulator by kicking the beam. A GENESIS simulation is overlaid (blue curve) using the beam parameters indicated. From this measurement, a gain length of $3.3 \mathrm{~m}$ is obtained. An image of the X-ray beam spot is shown in the inset (spot size $140 \mu \mathrm{m} \mathrm{rms)} \mathrm{(Reprinted} \mathrm{from} \mathrm{Refs.} 39$ and 97.)
FLASH (Fig. 13) shows that the SASE amplification process is well understood and simulation tools are well advanced.

From the LCLS gain curve, a power gain length of $L_{\mathrm{g}}=3.3 \mathrm{~m}$ is obtained. With the data given in [38], the 1D power gain length is calculated to be $L_{\mathrm{g} 0}=2.3 \mathrm{~m}$, showing large corrections to the 1D model. Saturation is obtained at approximately $z=$ $60 \mathrm{~m}$ hence after 18 gain lengths. This is again in good agreement with Eq. (29).

A more realistic picture of the high-gain amplification process is usually obtained using full 3D simulation codes like GINGER [52], GENESIS [53], or FAST [54]. Many aspects neglected by the 1D model are taken into account: for instance, the transverse extension and diffraction of the light wave, and important electron bunch parameters like emittance, energy spread, and short bunch length.

\section{Statistical Properties of SASE Radiation}

The stochastic nature of the shot noise radiation selfseeding the amplification process is responsible for the intrinsic fluctuation of the energy and wavelength spectra of the amplified FEL radiation.

Figure 7 shows an example of single shot spectra measured at FLASH. The single shot spectra vary in center wavelength and shape. The bold curve is an averaged spectrum over 300 shots. The average spectrum is well reproduced by a 3D simulation.

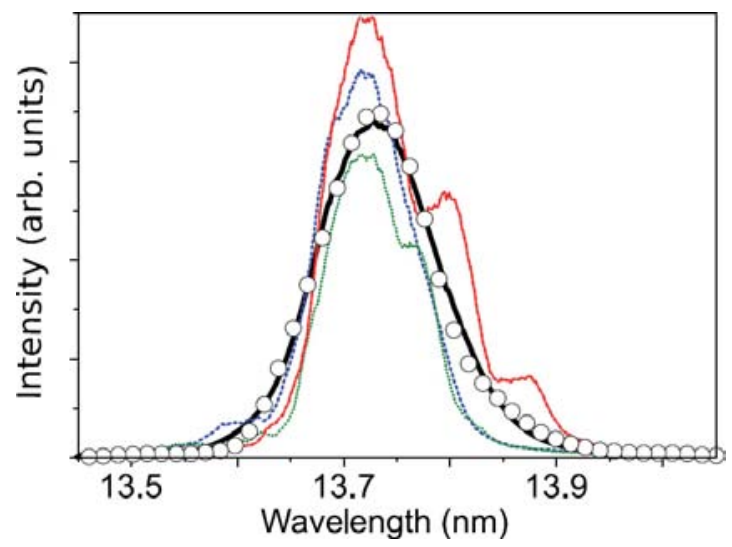

Fig. 7. Measured single shot spectra at FLASH. The bold line shows an averaged spectrum over 300 shots. The spectra are obtained in saturation at a single pulse energy of $40 \mu \mathrm{J}$. The circles indicate a simulation of the averaged spectrum with the 3D code FAST [54]. (Adapted from Ref. 32.) 
The single shot spectra mostly show a single spike; sometimes, two or three spikes are visible. Because the spectrum is simply a Fourier transform of the temporal structure, the temporal profile of the radiation pulses also consists mostly of a single spike. From time to time, also in the temporal profile, two or more spikes appear.

\subsection{Coherence time and pulse duration}

An important quantity in this context is the coherence time. This is the time over which a correlation in the radiation field exists.

Following the approach of Saldin et al. [67] and Schmüser et al. [43], the coherence time can be calculated from the first-order correlation function using the solution of the radiation field including a finite bandwidth $\sigma_{\omega}(z)$ [Eqs. (23) and (24)]:

$$
\tau_{\mathrm{coh}}(z)=\frac{\sqrt{\pi}}{\sigma_{\omega}(z)}=\frac{\sqrt{\pi}}{3 \sqrt{2} \omega_{\mathrm{s}} \rho_{\mathrm{fel}}} \sqrt{\frac{z}{L_{\mathrm{g} 0}}} .
$$

For FLASH, lasing with $13.7 \mathrm{~nm}$ in saturation, the coherence time is estimated with experimental data using Eq. (30): $\tau_{\text {coh }}=4.2 \pm 0.5$ fs [32].

A direct measurement of the coherence time has been carried out at FLASH with a split-and-delay autocorrelation experiment. The coherence time at $24 \mathrm{~nm}$ derived from the single shot interference pattern is $\tau_{\text {coh }}=5.1 \mathrm{fs}(\mathrm{rms})$ [65].

The same group used the autocorrelator together with two-photon double ionization of $\mathrm{He}$ as a nonlinear medium to measure the temporal length of the FEL pulse. Figure 8 shows the time-resolved yield of double ionized He [65]. From a fit to the data, a pulse duration of $\tau_{\mathrm{s}}=29 \pm 5 \mathrm{fs}$ has been derived. The plot also shows (as a dashed line) the spike structure of the FEL pulse in the time domain.

Another measurement of the pulse duration at FLASH at a wavelength of $13.5 \mathrm{~nm}$ yielded an rms pulse duration of $\tau_{\mathrm{s}}=15 \pm 3 \mathrm{fs}$ [66]. This time a streak camera technique with $\mathrm{THz}$ radiation from the FLASH THz undulator was used. Also, this experiment observed from time to time spikes in the time domain.

\subsection{Statistical mode analysis}

The spikes that appear in the frequency domain obviously have their origin in wave packets that are separated in time.

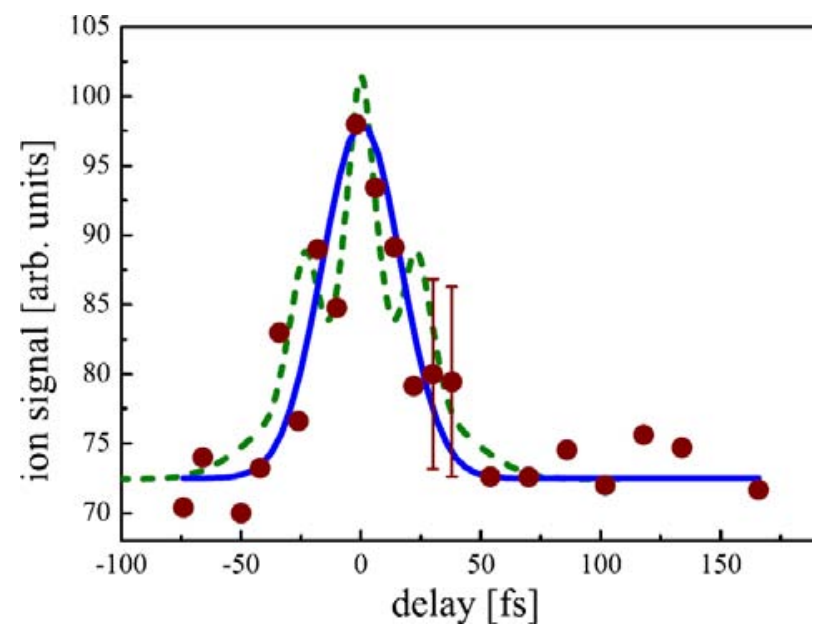

Fig. 8. Time-resolved double ionization of He (dots). The solid line is a Gaussian fit to the data with a width of $39 \mathrm{fs}$ FWHM. This yields a pulse duration of $\tau_{\mathrm{s}}=29 \pm 5 \mathrm{fs}$. The dashed line represents a three-pulse structure with temporal separations of the side peaks by 12 and $40 \mathrm{fs}$ with an added chirp of $50 \mathrm{fs}^{2}$. (Reprinted from Refs. 65 and 98.)

Because the pulse duration is longer than the coherence time, more than one wave packet may exist in the pulse. The average number of wave packets or spikes within the spectra is

$$
M=\frac{\tau_{\mathrm{s}}}{\tau_{\mathrm{coh}}} .
$$

Since the SASE FEL radiation has random amplitudes and phases in time and space, it can be described using statistical optics. During the exponential growth, the radiation pulse energy is fluctuating according to a gamma distribution $p(W)$ [67]:

$$
p(W)=\frac{M^{M}}{\Gamma(M)}\left(\frac{W}{\langle W\rangle}\right)^{M-1} \frac{1}{\langle W\rangle} \exp \left(-M \frac{W}{\langle W\rangle}\right) .
$$

$W$ is the energy of the individual pulse, and $\langle W\rangle$ the average energy of all SASE pulses considered in the measurement. $\Gamma(M)$ is the gamma function with the argument $M . M$ is the inverse of the squared normalized variance of the energy $\sigma_{W, \mathrm{n}}$ :

$$
\frac{1}{M}=\sigma_{W, \mathrm{n}}^{2}=\frac{\left\langle(W-\langle W\rangle)^{2}\right\rangle}{\langle W\rangle^{2}} .
$$

The parameter $M$ corresponds to the number of transverse and longitudinal optical modes, which is in fact identical to the number of longitudinal modes or spikes in the wavelength spectra, since the radiation close to saturation is transversely coherent. 

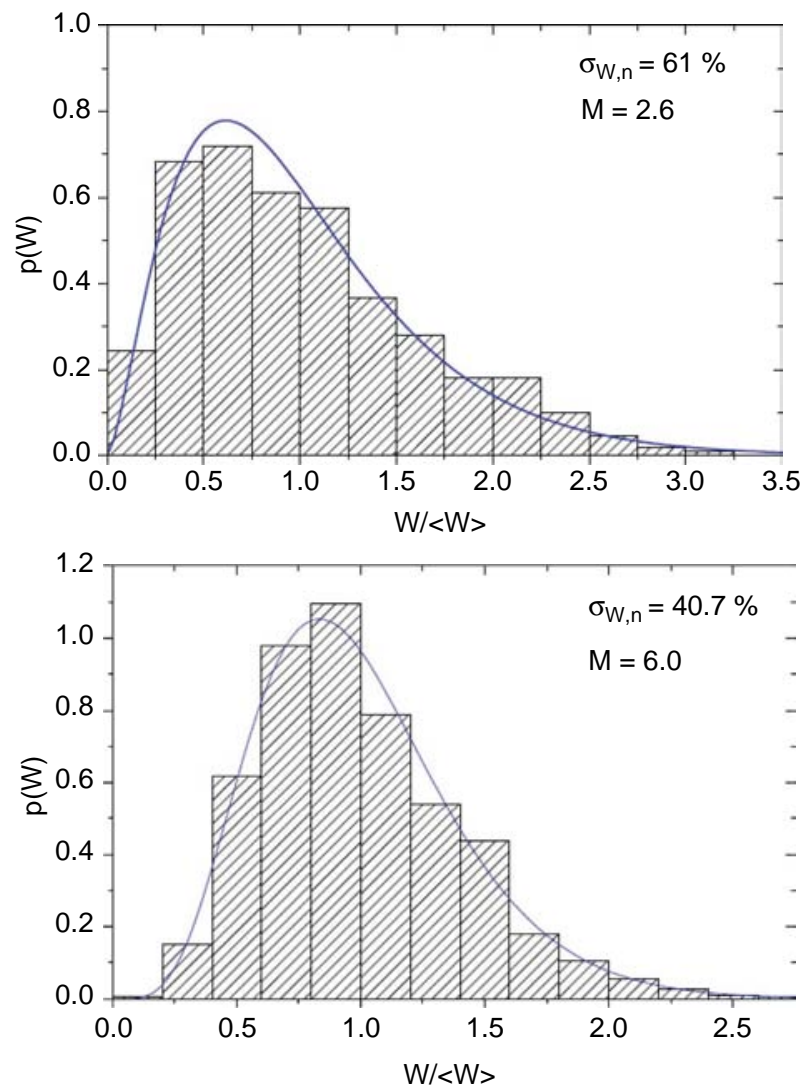

Fig. 9. Histograms of the probability distribution of the measured single pulse energies of several hundred pulses for different settings of the electron bunch length. The SASE pulses are observed at FLASH at high gain, but still in the exponential regime, just before saturation. The wavelength is $97 \mathrm{~nm}$. The upper plot is for a machine setting with short electron bunches; the lower plot is for long bunches. The solid curves represent gamma distributions according to Eq. (32), yielding a mode number $M=2.6$ for short and $M=6$ for long bunches. $M$ is calculated from the standard deviation of each distribution. The gamma distribution is not a fit; it is directly calculated from the data and normalized to the total energy of the data histogram. (Adapted from Refs. 30 and 95.)

As an example for the validity of this approach, Fig. 9 shows two distributions of the pulse energy of several hundred FEL pulses measured at FLASH with a wavelength of $97 \mathrm{~nm}$ for different electron pulse durations [30]. The mode number is calculated from the standard deviation of the energy distribution according to Eq. (33). The solid line overlaid on the energy distribution histogram is directly calculated from the data according to Eq. (32), not a fit. The calculated gamma distribution is normalized to the total energy of the histogram (sum over all bins). For long electron bunches, the mode number is $M=6.0$; for short bunches, $M=2.6$.
This result agrees remarkably well with the measurement of the spectra for the long and the short electron pulse case, as shown in Fig. 11. The number of spikes in the spectra corresponds to the mode number calculated from the energy distribution.

Convincing is also the change in the distribution when a single longitudinal mode is selected with a narrow-band monochromator. With only one mode present, the distribution approaches a negative exponential with the mode number $M=1$, calculated from the normalized standard deviation of the measured energy distribution (Fig. 10).

\subsection{Transverse coherence}

Similar to optical lasers, the fundamental Gaussian transverse mode has its highest intensity near the beam axis, while all other high-order modes have a larger transverse extent. Therefore, during the amplification process, the fundamental mode exhibits a larger amplification and will win the mode competition. Approaching saturation, the fundamental mode dominates and the radiation will have a high degree of transverse coherence. Measurements at FLASH at a wavelength of $13.7 \mathrm{~nm}$ with a double-slit systems show an almost-full transverse coherence [68]. The double-slit measurement demonstrates that the degree of coherence is similar for the horizontal and the vertical direction, and that the coherence length

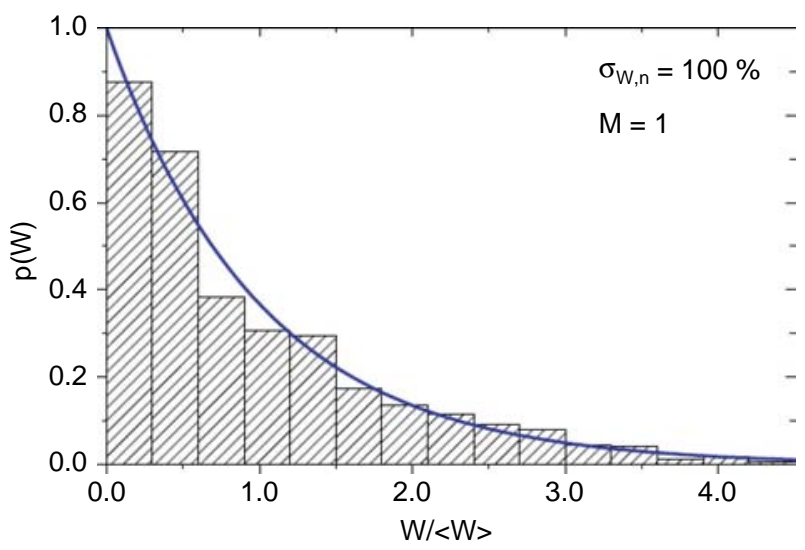

Fig. 10. Histograms of the probability distribution of the measured single pulse energies of several hundred pulses. The radiation pulses have passed a narrow-band monochromator. The curve shows the gamma distribution according to Eq. (32). As expected for monochromatic radiation, the distribution is a negative exponential and yields $M=1$. (Adapted from Refs. 30 and 95.) 


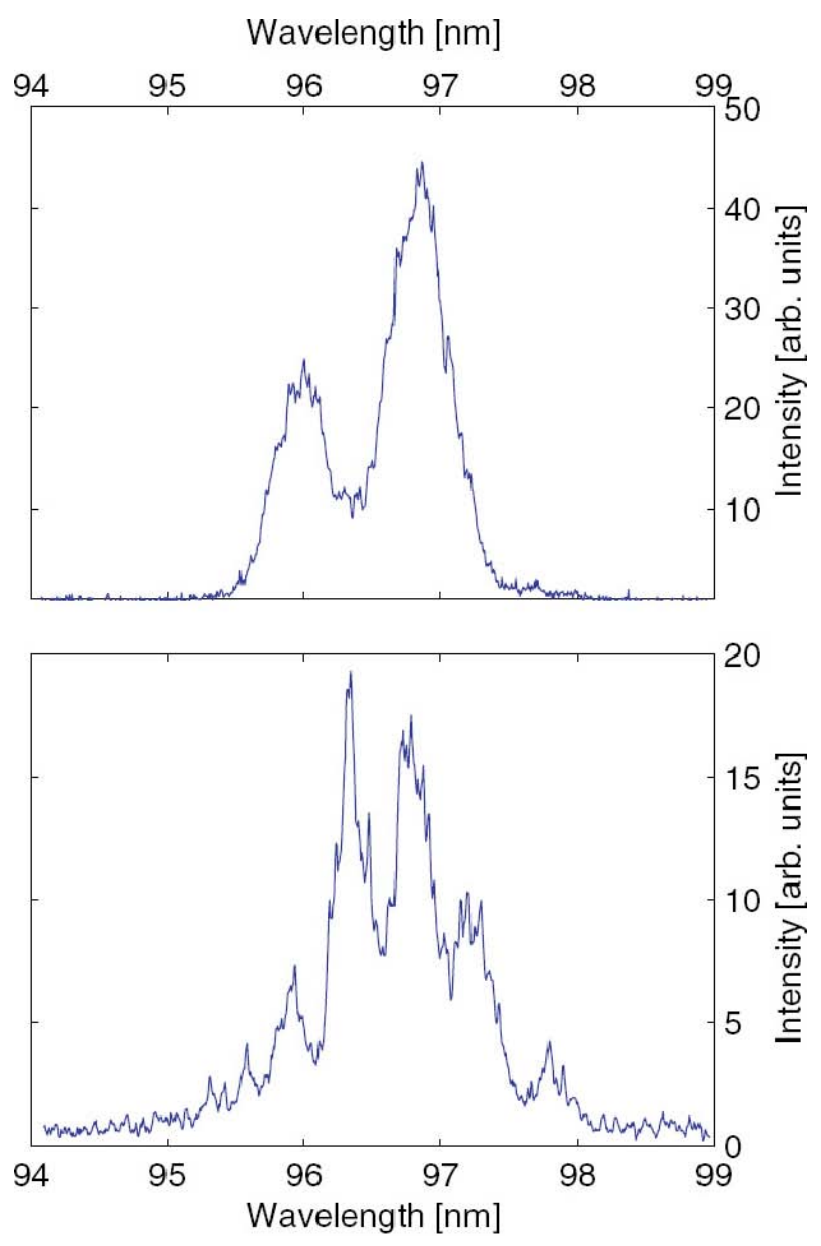

Fig. 11. Spectra measured at FLASH with short (top) and long (bottom) electron bunches. The number of spikes corresponds well to the mode number calculated from the standard deviation of the energy fluctuations, as shown in Fig. 9. The average number of spikes obtained from the statistical analysis is 2.6 in the short pulse case (upper plot), and 6 in the long pulse case (lower plot). (Adapted from Refs. 30 and 95.)

scale is about $300 \pm 15 \mu \mathrm{m}$ at a distance of $20 \mathrm{~m}$ downstream of the undulator (Fig. 12).

It is worth mentioning that, in deep saturation, higher modes gain in energy with respect to the fundamental mode, with the consequence of a reduced transverse coherence. A more detailed discussion can be found in [69].

\section{Requirements for Electron Bunch Parameters}

For the realization of an FEL, the requirements for electron beam parameters to achieve a reasonably small gain length are of the utmost importance.

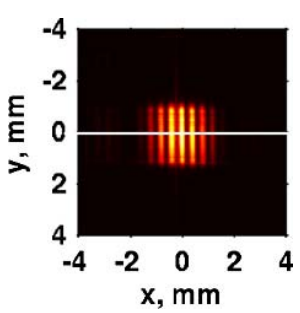

(a)

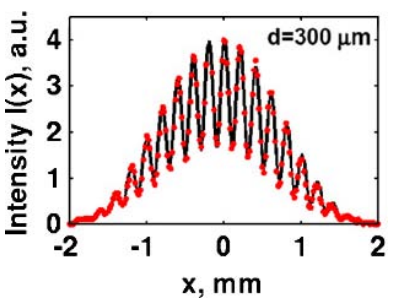

(c)

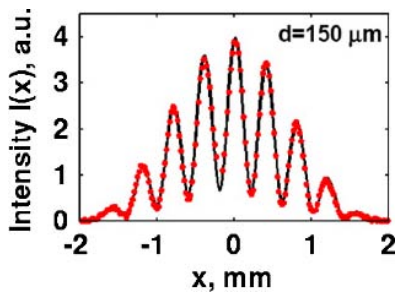

(b)

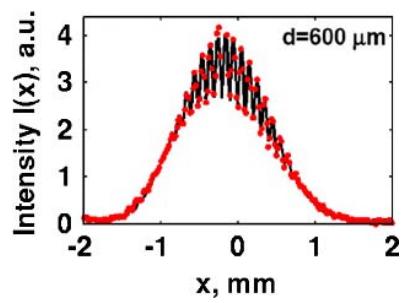

(d)
Fig. 12. Double-slit experiment at FLASH at a wavelength of $13.7 \mathrm{~nm}$. (a) A typical image of the diffraction pattern with vertical slits of $150 \mu \mathrm{m}$ separation. (b)-(d) Results of a fit (solid lines) to experimental data (points) for different slit separations: $d=150 \mu \mathrm{m}$ (b), $d=300 \mu \mathrm{m}$ (c), and $d=600 \mu \mathrm{m}(\mathrm{d})$. (Adapted from Refs. 68 and 99.)

Generally speaking, considering more realistic beam parameters using a full 3D model, the real gain length $L_{\mathrm{g}}$ increases compared to the gain length obtained with the 1D model: $L_{\mathrm{g}}>L_{\mathrm{g} 0}$. The expected gain length has important consequences for the layout of the undulator system. Therefore, requirements for electron beam properties must be carefully studied when designing an SASE FEL.

\subsection{Beam current}

To estimate the required electron density for a reasonable gain length of $L_{\mathrm{g} 0} \approx 1 \mathrm{~m}$, we use Eq. (17) together with Eq. (14) and assume typical undulator parameters, beam size, and an energy of $1 \mathrm{GeV}$ (see Subsec. 4.2.1):

$$
L_{\mathrm{g} 0}=8.4 \cdot 10^{6} \frac{1}{\sqrt[3]{n_{\mathrm{e}}}} .
$$

Using the expression of $n_{\mathrm{e}}$ in terms of peak current [Eq. (10)] and bunch rms size $\sigma_{\mathrm{b}}$,

$$
n_{\mathrm{e}}=\frac{1}{e c} \cdot \frac{I_{0}}{2 \pi \sigma_{\mathrm{b}}^{2}},
$$

we obtain

$$
I_{0}=1.2[\mathrm{kA}]\left[\mathrm{m}^{3}\right] \frac{1}{L_{\mathrm{g} 0}^{3}} .
$$


It is evident that for a power gain length on the order of $1 \mathrm{~m}$, a peak current in the $\mathrm{kA}$ range is required.

It should be noted that, in practice, the electron density $n_{\mathrm{e}}$ is determined by the bunch or peak current $I_{0}$, and to a lesser extent by the transverse beam size $\sigma_{\mathrm{b}}$, which is typically in the range of $50-100 \mu \mathrm{m}$.

Let us discuss the effect of the space charge parameter $k_{p}$ on the gain length. From Eqs. (15) and (19) we know that

$$
k_{p} \propto \sqrt{n_{\mathrm{e}}} \propto \sqrt{I_{0}} .
$$

Solving Eq. (11) taking the space charge parameter $k_{p}$ into account leads to the conclusion that for

$$
k_{p}<0.5 \Gamma
$$

the increase in gain length is still moderate and on the order of $10 \%$ [ 43$]$.

Indeed, this is the case for X-ray FELs. For our typical parameters, we obtain the expression

$$
\frac{k_{p}}{\Gamma} \approx 0.04\left(I_{0}[A]\right)^{1 / 6} .
$$

Since the bunch current enters only with the power to $1 / 6$, a ratio of $k_{p} / \Gamma=0.5$ is obtained for unrealistic high peak currents of $5 \mathrm{MA}$. Only for very low beam energies of less than about $100 \mathrm{MeV}$ we do need to consider the effect of the space charge parameter. For FLASH, $k_{p}=0.14 \Gamma$, resulting in an increase of gain length by $1 \%$ only.

\subsection{Energy spread}

Owing to the narrow bandwidth of the high-gain FEL, an energy spread of the beam $\sigma_{W}$ has a larger effect on the gain length. Only if all electrons have the same energy $W_{\mathrm{r}}$ is the power gain length close to the real gain length $L_{\mathrm{g}}$. Calculations show that for $\sigma_{W} / W_{\mathrm{r}}=0.5 \rho_{\mathrm{fel}}$, the gain length is already increased by $25 \%$ [43]. This defines a reasonable upper limit:

$$
\frac{\sigma_{W}}{W_{\mathrm{r}}}<0.5 \rho_{\mathrm{fel}} .
$$

With a typical FEL parameter of $\rho_{\mathrm{fel}}=1.6$. $10^{-3}$, the energy spread needs to be kept well below $1 \cdot 10^{-3}$.

An important consideration is a possible spacecharge-induced energy chirp $d W / d t$ along the bunch $[55,56]$. The effect of the energy chirp on the gain length starts to play a significant role when the relative energy change $d W / W$ acquired within one coherence length $\tau_{\text {coh }}$ becomes comparable to the FEL parameter:

$$
\frac{\tau_{\mathrm{coh}}}{d t} \frac{d W}{W}=\frac{\tau_{\mathrm{coh}}}{W} \frac{d W}{d t}<0.5 \rho_{\mathrm{fel}} .
$$

As reported in [32], space charge effects induce a considerable energy chirp on the lasing part of the bunch at FLASH $(\lambda=13.7 \mathrm{~nm})$. The parameter defined in Eq. (41) amounts to $1.5 \rho_{\text {fel }}$, much larger than the limit of $0.5 \rho_{\mathrm{fel}}$. Indeed, the measured power gain length is, with $L_{\mathrm{g}}=1.25 \pm 0.15 \mathrm{~m}$, about a factor of 2.5 higher than calculated from the $1 \mathrm{D}$ theory. The large energy spread or chirp has also the effect of increasing the width of the frequency spectrum.

\subsection{Emittance}

Besides the requirements on peak current and energy spread, achieving and maintaining an overlap of the electron beam with the amplified radiation field in long undulator sections is of the utmost importance.

\subsubsection{Emittance definition}

Important parameters in this context are the electron beam emittance $\epsilon$ and its equivalent, the diffraction limit of the amplified radiation $\lambda_{\mathrm{s}} / \pi$. For both, the electron beam and the radiation, this quantity is as usual defined by the product of the size and divergence with an additional correlation term, if required.

The emittance of an electron bunch is usually defined in terms of measurable quantities, which is also applicable in simulation codes [58]:

$$
\epsilon=\frac{1}{\bar{p}_{z}} \sqrt{\left\langle x^{2}\right\rangle\left\langle p_{x}^{2}\right\rangle-\left\langle x p_{x}\right\rangle^{2}}
$$

with $x$ being the Cartesian transverse horizontal coordinate of the electron, and $p_{x}$ the momentum component in the horizontal direction. An equivalent equation holds for the vertical coordinate $y$. The angular brackets define the second central moment of the distributions. Note that $\bar{p}_{z}$ is the average longitudinal momentum of the electrons. The coordinate $z$ is in the direction of acceleration. The emittance defined in this way is an rms emittance.

In the following, we always assume round beams with equal transverse dimensions $\sigma_{\mathrm{b}}=\sigma_{x}=\sigma_{y}$ and use the indices $x$ or $b$, representing both of the transverse coordinates $x$ and $y$. 
In experiments, the terms with transverse momenta $p_{x}$ are often estimated by the measured beam divergence, for instance $\sigma_{x}^{\prime 2}=\left\langle\left(p_{x} / p_{z}\right)^{2}\right\rangle$. The rms beam size is $\sigma_{x}^{2}=\left\langle x^{2}\right\rangle$.

The emittance as defined above is equivalent to the Courant-Snyder invariant $W=\gamma x^{2}+$ $2 \alpha x x^{\prime}+\beta x^{\prime 2}$, defined in [59]; $\alpha, \beta$, and $\gamma$ are the Twiss parameters, $x^{\prime}=d x / d z$, with $z$ the direction of motion. The relation between emittance and beam size in terms of the beta function $\beta(z)$ is given by

$$
\sigma_{x}^{2}(z)=\epsilon \beta_{x}(z)
$$

As an example, a beam size of $\sigma_{x}=100 \mu \mathrm{m}$ with a beta function $\beta_{x}(z)$ of $10 \mathrm{~m}$ is achieved with an emittance of $\epsilon=1 \mathrm{~nm}$.

Equation (42) shows that the emittance is adiabatically damped with the average longitudinal beam momentum $\bar{p}_{z}$. Therefore, it is convenient to define a normalized emittance $\epsilon_{\mathrm{n}}$ :

$$
\epsilon_{\mathrm{n}}=\frac{\bar{p}_{z}}{m_{\mathrm{e}} c} \epsilon \approx \gamma \epsilon
$$

\subsubsection{Beta function and gain length}

A good overlap between electron beam and radiation is usually obtained by adapting the electron beam size and divergence to the size and divergence of the radiation.

Usually, the principle of strong focusing $[47,57]$ is applied: quadrupoles are placed between undulator segments of suitable length to form for instance a FODO lattice. Beta functions between 10 and $30 \mathrm{~m}$ are realized. Typically, the lattice period is smaller than the beta function. The electron beam optics also takes into account the weak focusing in the vertical direction of the undulator magnets.

The betatron oscillation has an effect on the lasing process. It introduces into the already-discussed oscillation induced by the undulator field an additional transverse velocity component. For the FEL process, this translates into an effective reduction of the beam energy by $d \gamma_{\text {eff }}=-\gamma^{3} \epsilon / \beta_{x}$ and, in addition, into an effective smearing of the beam energy by $\sigma_{\eta}$,eff $=\gamma^{2} \epsilon / \beta_{x}$. We neglect the variation of the beta function along the undulator and use an average beta function: $\beta(z) \approx \beta_{\mathrm{av}}$.

Adding both independent effects in quadrature and using the condition for an acceptable energy spread being $0.5 \rho_{\mathrm{fel}}$, Eq. (40), we obtain the following condition for the transverse beam emittance:

$$
\epsilon<\frac{\beta_{\mathrm{av}}}{2 \sqrt{2} \gamma^{2}} \rho_{\mathrm{fel}} .
$$

As an example, for an average beta function of $5 \mathrm{~m}$ along the undulator and a beam energy of $1 \mathrm{GeV}$ with a typical FEL parameter of $\rho_{\text {fel }}=2 \cdot 10^{-3}$, we obtain a normalized emittance limit of $\epsilon_{\mathrm{n}}<1.8 \mu \mathrm{m}$. Taking LCLS parameters [38], we obtain a limit of $\epsilon_{n}<0.3 \mu \mathrm{m}$ with $\beta_{\mathrm{av}}=30 \mathrm{~m}$. (We use the calculated FEL parameter $\rho_{\text {fel }}=7.3 \cdot 10^{-4}$; the LCLS beam energy is $13.6 \mathrm{GeV}$.)

It is interesting to note that for the normalized emittance $\epsilon_{\mathrm{n}}$ the condition (45) can be expressed in terms of the beam size $\sigma_{x}$ and the FEL parameter $\rho_{\text {fel }}$ only:

$$
\epsilon_{\mathrm{n}}<\sigma_{x} \sqrt{\frac{\rho_{\mathrm{fel}}}{2 \sqrt{2}}} .
$$

If we chose an average beam size along the undulator of $\sigma_{x}=80 \mu \mathrm{m}$, the normalized emittance limit is $2.1 \mu \mathrm{m}\left(\rho_{\mathrm{fel}}=0.002\right)$. Since the FEL parameter scales with $1 / \gamma$, the emittance limit scales with $1 / \sqrt{\gamma}$ and has only a weak dependence on the energy. This makes it possible to adjust the condition for lasing by choosing the appropriate beam size, eventually relaxing the requirements on the beam emittance of the electron source. However, one has to consider that large beam sizes reduce the electron density in the bunch and thus increase the gain length since

$$
L_{\mathrm{g} 0} \propto \frac{1}{\sqrt[3]{n_{\mathrm{e}}}} .
$$

\subsubsection{Diffraction of the light wave}

To discuss the overlap of the electron beam with the light wave, we need to take into account that for a diffraction-limited laser beam, the product of the Gaussian waist size $w(z=0)=w_{0}=2 \sigma_{l, 0}$ and halfangle divergence $\theta$ with respect to the waist $w(z \rightarrow$ $\infty)$ is a constant given by the light wavelength $\lambda-$ very similar to the electron beam emittance:

$$
\theta w_{0}=\frac{\lambda}{\pi} .
$$

As an example, for a wavelength of $50 \mathrm{~nm}$ and a laser beam waist size of $\sigma_{l, 0}=100 \mu \mathrm{m}$, its size is doubled after $2.5 \mathrm{~m}$. This length is also known as the 
Rayleigh length:

$$
z_{\mathrm{R}}=\frac{\pi w_{0}^{2}}{\lambda}=\frac{4 \pi \sigma_{l, 0}^{2}}{\lambda} .
$$

As we know from a typical gain length of about $1 \mathrm{~m}$, the undulator often needs to be much longer than the Rayleigh length of $2.5 \mathrm{~m}$. This does not necessarily mean that after a distance $z>z_{\mathrm{R}}$ the light wave expands much faster than the electron beam and light is lost. We have to take into account that for all positions along the undulator, the wave is exponentially amplified and thus is freshly created along the undulator with a waist $w_{0}$ of twice the electron beam size $\sigma_{x}$ until saturation is reached. This effect is well known from conventional lasers and is often called "gain guiding."

Indeed, with a too-small Rayleigh length, photons will be lost during the amplification process. This is taken into account in 3D simulations. See [60] for a discussion on this subject.

The effect is not so dramatic for shorter wavelengths: for $\lambda=5 \mathrm{~nm}$, the Rayleigh length is already $25 \mathrm{~m}$ for $\sigma_{l, 0}=100 \mu \mathrm{m}$. The length of the FLASH undulator system is $27 \mathrm{~m}$.

Gain guiding is beneficial for long undulator systems for X-ray FELs, since it permits slow deviations of the electron beam orbit and facilitates undulator positioning. The pointing of the FEL beam to the experiments will be determined by the alignment accuracy of the last undulator segments, and to a lesser extent by the initial orbit of the electron beam.

To summarize, the loss due to diffraction effects is small if the Rayleigh length is larger than the gain length:

$$
z_{\mathrm{R}}>L_{\mathrm{g} 0} .
$$

Fortunately, this condition is usually fulfilled, as illustrated by the following example. For an emittance of $\epsilon<0.9 \mathrm{~nm}$ fulfilling the criterion of Eq. (45), the average beam size along the undulator is $70 \mu \mathrm{m}$. We assume a beta function of $\beta_{\mathrm{av}}=5 \mathrm{~m}$, and a beam energy of $1 \mathrm{GeV}$. Taking the FLASH undulator, a beam energy of $1 \mathrm{GeV}$ would correspond to a laser wavelength of $6.3 \mathrm{~nm}$. For a laser beam size equal to the electron beam size, the Rayleigh length for this wavelength is $z_{\mathrm{R}}=10 \mathrm{~m}$, much longer than the gain length of $L_{\mathrm{g} 0}=0.7 \mathrm{~m}$. Also, at LCLS [38], the Rayleigh length of $37 \mathrm{~m}\left(\lambda_{\mathrm{s}}=1.5 \AA\right)$ is larger than the 1D gain length of $L_{\mathrm{g} 0}=1.9 \mathrm{~m}$.

\subsubsection{Overlap of the electron beam with the light wave}

A good criterion for a perfect overlap of electron and laser beam motivated by a simple physical argument is derived equaling the beam emittance (42) with the equivalent laser beam emittance, the diffraction limit of the radiation (48):

$$
\epsilon \leq \sigma_{l, 0} \theta=\frac{\lambda_{\mathrm{s}}}{4 \pi}
$$

In contrast to the criterion for the normalized emittance (46) derived from betatron oscillation of the electron beam, the limit (51) now scales for a given undulator with $1 / \gamma$ :

$$
\epsilon_{\mathrm{n}} \leq \frac{\lambda_{\mathrm{u}}}{8 \pi \gamma}\left(1+\frac{K^{2}}{2}\right) \propto \frac{1}{\gamma}
$$

The scaling holds under the assumption that the choice of undulator parameters does not change significantly for different beam energies. This is usually the case for X-ray FELs. As an example, the normalized emittance limit according to Eq. (52) is $\epsilon_{\mathrm{n}}<1 \mu \mathrm{m}$ for the case of lasing at $6.3 \mathrm{~nm}$ with $1 \mathrm{GeV}$ at FLASH. The limit using LCLS parameters from [38] is $\epsilon_{\mathrm{n}}<0.3 \mu \mathrm{m}$ (lasing at $1.5 \AA$ with $13.6 \mathrm{GeV})$.

\subsection{Parametrization of the gain length}

A simple analytically valid formula for the gain length including all 3D effects does not exist. Based on the exact solution and variational approximation of $3 \mathrm{D}$ eigenmodes in high-gain FELs, Xie proposed the following parametrization [61]:

$$
L_{\mathrm{g}}=L_{\mathrm{g} 0}(1+\Lambda) .
$$

The parameter $\Lambda$ is determined by interpolating the variational solutions. It contains three dimensionless parameters expressing the dependence of the gain length on the energy spread, on the emittance and betatron oscillations of the electron beam, and on the diffraction of the light wave - very similar to the discussion above.

Employing FLASH parameters for lasing at $13.7 \mathrm{~nm}$ from [32] $\left(\epsilon_{\mathrm{n}}=1.4 \mu \mathrm{m}, \sigma_{\mathrm{b}}=80 \mu \mathrm{m}, L_{\mathrm{g}, 0}=\right.$ $0.52 \mathrm{~m}$ ), we can use the parametrization to estimate the energy spread of the lasing spike, which is difficult to measure. An energy spread of $0.2 \%$ then leads to a correction of $\Lambda=1.4$, and to a $3 \mathrm{D}$ gain length of 


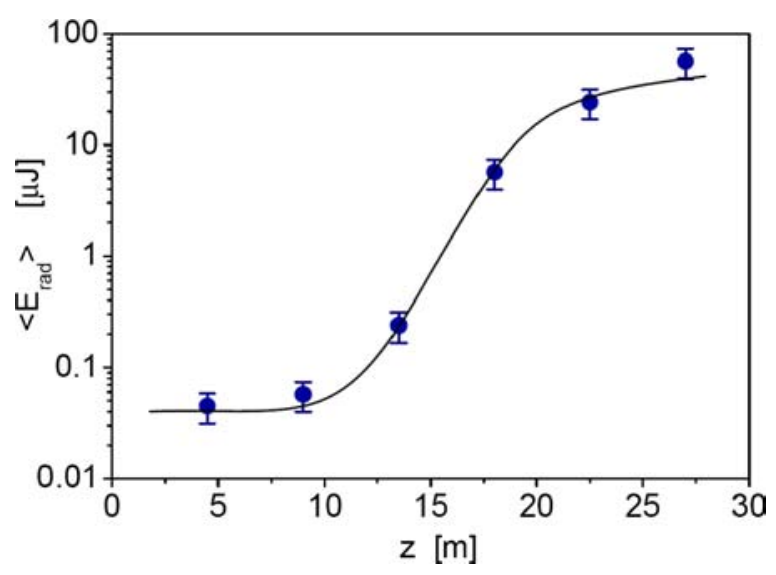

Fig. 13. Measured FEL pulse energy along the FLASH undulator at a wavelength of $13.7 \mathrm{~nm}$ [32]. For this measurement, the electron beam is kicked off the optimal trajectory at various positions along the undulator to suppress lasing. The measured energy includes the spontaneous radiation. From this data, the power gain length is estimated to be $L_{\mathrm{g} 0}=$ $1.25 \pm 0.15$ m. (Adapted from Refs. 32, 96.)

$1.26 \mathrm{~m}$ - matching the measured power gain length of $L_{\mathrm{g}}=1.25 \mathrm{~m}$.

A quite different parametrization has been proposed by Saldin et al. [62]. They evaluated an expression for the gain length which explicitly includes beam and undulator parameters. Their formula is not universal, but it provides a good accuracy that is better than $5 \%$ in the parameter range of soft and hard X-ray FELs. The power gain length is written in the form

$$
L_{\mathrm{g}}=\frac{1.67}{\sqrt{2}} \sqrt{\frac{I_{\mathrm{A}}}{I_{0}}} \frac{\left(\epsilon_{\mathrm{n}} \lambda_{\mathrm{u}}\right)^{5 / 6}}{\lambda_{\mathrm{s}}^{2 / 3}} \frac{\left(1+K^{2} / 2\right)^{1 / 3}}{K_{\mathrm{JJ}}} \cdot(1+\delta),
$$

with the Alfvén current $I_{\mathrm{A}}=4 \pi \epsilon_{\mathrm{o}} m_{\mathrm{e}} c^{3} / e=17.1 \mathrm{kA}$ and

$$
\delta=262 \frac{I_{\mathrm{A}}}{I_{0}} \frac{\epsilon_{\mathrm{n}}^{5 / 4}}{\lambda_{\mathrm{s}}^{1 / 8} \lambda_{\mathrm{u}}^{9 / 8}} \frac{\left(\sigma_{W} / m_{\mathrm{e}} c^{2}\right)^{2}}{K_{\mathrm{JJ}}^{2}\left(1+K^{2} / 2\right)^{1 / 8}} .
$$

This approximation is valid when the average beta function is optimized to achieve the smallest gain length for a given setup. The optimized beta function can be expressed in terms of beam and undulator parameters [62]:

$$
\beta_{\mathrm{opt}}=11.2 \sqrt{2} \sqrt{\frac{I_{\mathrm{A}}}{I_{0}}} \frac{\epsilon_{\mathrm{n}}^{3 / 2} \sqrt{\lambda_{\mathrm{u}}}}{\lambda_{\mathrm{s}} K_{\mathrm{JJ}}}(1+8 \delta)^{-1 / 3} .
$$

\subsection{Saturation power}

The parametrization by Kim and Xie [63] approximates the saturation power by

$$
P_{\text {sat }} \approx 1.6 P_{\text {beam }} \rho_{\text {fel }}\left(\frac{L_{\mathrm{g} 0}}{L_{\mathrm{g}}}\right)^{2},
$$

where $P_{\text {beam }}$ is the electron beam power:

$$
P_{\text {beam }}=\frac{\gamma_{\mathrm{r}} m_{\mathrm{e}} c^{2}}{e} I_{0} .
$$

The bunch current $I_{0}$ has been defined in Eq. (10) as the total charge $Q=e N_{\mathrm{e}}$ divided by the bunch duration $T: I_{0}=e N_{\mathrm{e}} / T$.

With typical parameters for FLASH, the FEL efficiency $P_{\text {sat }} / P_{\text {beam }}$ is almost $0.1 \%$. Since the beam power is $2.5 \mathrm{TW}$, this approximation leads to a saturation power of $P_{\text {sat }}=2.3 \mathrm{GW}$.

Figure 14 shows the dependence of the saturation power on emittance, energy spread, and peak current according to Eq. (57) using the parametrization of Xie, Eq. (53). Only one of the three parameters is varied for each curve. For the emittance scan, the average beam size in the undulator is kept constant and the beta function is adapted accordingly. This may lead to small beta functions for the larger emittance values, which may not be realistic in practice. The peak current is varied by changing the bunch length only, keeping the charge constant. The plot in Fig. 14 gives an impression of the electron beam properties required to drive a high-gain SASE FEL to saturation with peak powers at the GW level.

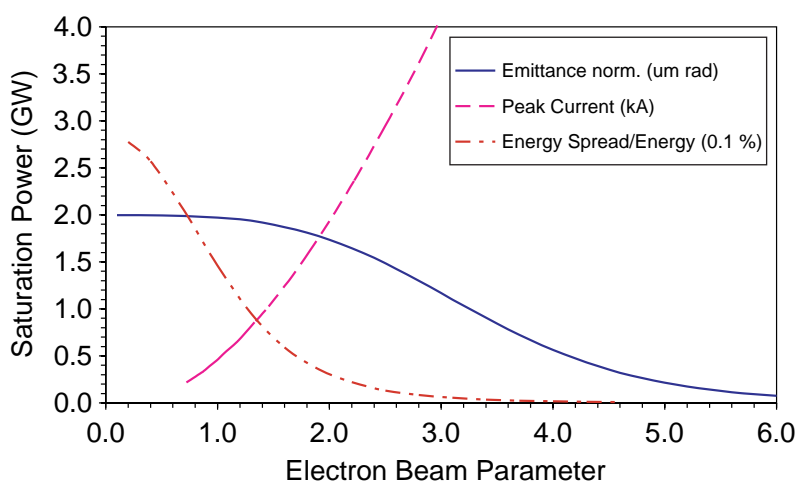

Fig. 14. Saturation power as a function of normalized emittance, relative energy spread, and peak current according to the approximation (57) using the parametrization of Xie, Eq. (53). 


\section{Brilliance}

One of the most striking and exciting properties of SASE radiation is its extreme brilliance compared to other light sources. Figure 1 shows the peak brilliance of soft and hard X-ray FELs compared to some third generation light sources with experimental data from FLASH and LCLS included.

The brilliance is defined as the photons flux $\dot{N}_{\text {ph }}$ within a certain spectral bandwidth $d \omega / \omega$ per unit area $A$ and unit solid angle $\Omega$ :

$$
B=\omega \frac{\dot{N}_{\mathrm{ph}}}{d \omega} \frac{1}{A \Omega} .
$$

The unit of the brilliance is photons $/\left(\mathrm{s} \mathrm{mm}^{2} \mathrm{mrad}^{2}\right.$ $0.1 \% \mathrm{bw}$ ). The notion "peak" refers to the brilliance of a single FEL pulse; in other words, $\dot{N}_{\mathrm{ph}}$ is the number of photons per pulse duration $T$. Sometimes, the average brilliance is used. Here $\dot{N}_{\text {ph }}$ is the number of photons per unit time. This is useful for multibunch FELs or storage-ring-based light sources, where many pulses per unit time are produced.

The area and solid angle are defined as rms values. Assuming a symmetric beam, we obtain $A=$ $2 \pi \sigma_{\mathrm{b}}^{2}$ and $\Omega=2 \pi \sigma_{\theta}^{2}$. For a high-gain FEL, the radiation has full transverse coherence, with the fundamental Gaussian mode dominating. Therefore, it is justified to use the diffraction limit (48): $\sigma_{\mathrm{b}} \sigma_{\theta}=$ $\lambda / 4 \pi$. Including this in Eq. (59), one obtains

$$
B_{\mathrm{fel}}=\omega \frac{d \dot{N}_{\mathrm{ph}}}{d \omega} \frac{1}{(\lambda / 2)^{2}} .
$$

The brilliance can be expressed in easily measurable quantities - the energy of the photon pulse, the wavelength, and the number of modes.

In the exponential gain regime and also close to saturation, the spectrum has a Gaussian shape leading to $\omega d \dot{N}_{\mathrm{ph}} / d \omega=\omega \dot{N}_{\mathrm{ph}} / \sqrt{2 \pi} \sigma_{\omega}$. Assuming a random flat longitudinal charge distribution with a total pulse duration $T$, we get $\dot{N}_{\mathrm{ph}}=N_{\mathrm{ph}} / T$. The brilliance now reads

$$
B_{\mathrm{fel}}=\frac{\omega N_{\mathrm{ph}}}{\sqrt{2 \pi} \sigma_{\omega} T} \frac{1}{(\lambda / 2)^{2}} .
$$

Including the coherence time $\tau_{\text {coh }}=\sqrt{\pi} / \sigma_{\omega}$ and using Eq. (31), we can express the brilliance with the number of modes $M=T / \tau_{\text {coh }}$ :

$$
B_{\mathrm{fel}}=\frac{4 \sqrt{2} c N_{\mathrm{ph}}}{\lambda^{3}} \frac{1}{M} .
$$

The number of photons is calculated by measuring the energy of a single pulse $W_{\mathrm{ph}}$ with
$N_{\mathrm{ph}}=\lambda / h c W_{\mathrm{ph}}$. It is determined by applying the statistical analysis discussed in Sec. 6. It is then simply the squared inverse normalized rms fluctuation of the pulse energy $M=1 / \sigma_{W, \mathrm{n}}^{2}$ [Eq. (33)]. $M$ now also includes possible transverse modes, which have initially been neglected.

Finally, we get a simple expression for the peak brilliance for high-amplification FELs:

$$
B_{\mathrm{fel}}=\frac{4 \sqrt{2}}{h} \frac{W_{\mathrm{ph}}}{\lambda^{2}} \sigma_{W, \mathrm{n}}^{2} .
$$

\section{The Electron Source}

As discussed in more detail in the previous sections, the requirements on the electron bunches can be summarized as follows: high peak current in the $\mathrm{kA}$ range with a transverse normalized emittance of around $1 \mu \mathrm{m}$, and an energy spread of $0.1 \%$ with an energy in the $\mathrm{GeV}$ range.

Where the electron source is concerned, the requirement on the emittance together with the charge on the order of $1 \mathrm{nC}$ needed to achieve high current densities is most challenging.

A technical solution is the RF gun introduced by Fraser and Sheffield in 1986 [70]. The electrons are produced in a strong accelerating field by a photocathode driven with a suitable laser system. Rapid acceleration reduces emittance growth, and laserinduced emission allows the production of picosecond short bunches from the source. This makes a classical bunching section superfluous.

A typical RF gun consists of a half-cell followed by one or more full cells. Guns have been built for many $\mathrm{RF}$ frequencies, the most common being the S-band $(\approx 3 \mathrm{GHz})$ or L-band $(1.3 \mathrm{GHz})$.

As an example, Fig. 15 shows a drawing of the FLASH RF gun.

The photocathode is placed at the center of the half-cell backplane. The drive laser power is adapted to the quantum yield of the photocathode. Laser systems with mode-locking techniques are used to synchronize the picosecond long laser pulses to the RF field to better than $1^{\circ}$ (in terms of the RF phase).

Photocathodes and laser systems are adapted to the specific requirement of the facility. Single shot FELs with a low repetition rate, like LCLS or SPARC, use $\mathrm{Cu}$ cathodes. The low quantum efficiency of $\mathrm{Cu}$ of $\approx 10^{-5} \cdots 10^{-4}$ needs a powerful laser system in the UV. However, single shot laser 


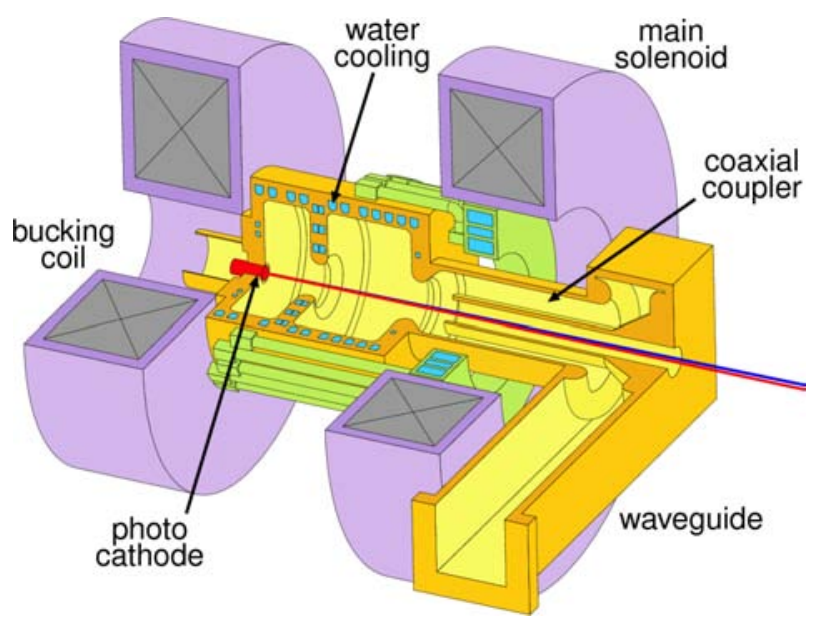

Fig. 15. Drawing of the FLASH RF gun. This is a $1.3 \mathrm{GHz}$ 1 and 1/2-cell copper structure powered by a $5 \mathrm{MW}$ klystron. The RF pulse duration is $850 \mu \mathrm{s}$, with a repetition rate of $10 \mathrm{~Hz}$. The water-cooling system is designed to remove $50 \mathrm{~kW}$ of heat. The photocathode is a thin layer of $\mathrm{Cs}_{2} \mathrm{Te}$. The laser beam enters at a small angle. The focusing solenoid is placed at the exit of the gun. An additional magnet close to the cathode compensates for the remaining field of the solenoid to zero. (Courtesy of M. Krasilnikov and E. Vogel, DESY.)

systems meeting the requirements of RF guns are commercially available.

For burst mode FELs like FLASH or the European XFEL, the situation is very different. The number of bunches per second is about a factor of 1000 larger than for today's single shot FELs. Since the average laser beam power of reasonable-sized laser systems is limited to a couple of watts, a cathode with a yield surpassing $\mathrm{Cu}$ by the same factor of 1000 is required.

FLASH uses a thin film of $\mathrm{Cs}_{2} \mathrm{Te}$ deposited on a Mo substrate. This type of cathode was introduced at the CLIC Test facility in 1993 [71] and is used now by many facilities around the world. The cathode is robust, with a high quantum efficiency of $>5 \%$ [72]. Under good ultrahigh-vacuum conditions of less than $10^{-10}$ mbar, the lifetime exceeds 100 days [73].

Suitable burst mode laser systems with an average power of a couple of watts have been built for FLASH by the Max-Born Institute, Berlin [74].

\subsection{Space charge forces}

Due to strong space charge forces at small electron energies, it is not possible to produce bunches with a peak current exceeding $100 \mathrm{~A}$ directly at the source. But even for relatively small charge densities, space charge forces may induce growth of the projected transverse emittance.

The radial force $F_{r}$ inside a cylindrical electron bunch with $N_{\mathrm{e}}$ electrons, a radius of $r_{\mathrm{b}}$ and a length $l_{\mathrm{b}}$ is given by the expression [43]

$$
F_{r}(r)=\frac{N_{\mathrm{e}} e^{2}}{2 \pi \epsilon_{\mathrm{o}} l_{\mathrm{b}}} \frac{r}{r_{\mathrm{b}}^{2}} \frac{1}{\gamma^{2}},
$$

with $r \leq r_{\mathrm{b}}$. The force has a defocusing effect and thus increases the projected emittance. It is important that the defocusing force is strongly reduced with beam energy $\approx 1 / \gamma^{2}$. Therefore, the beam must be accelerated as rapidly as possible in order to mitigate this effect.

For a cylindrical bunch, the defocusing force is linear with the radius (64). Carlsten proposed compensating for the space-charge-induced emittance growth by a simple solenoid lens with a field growing linear with $r$ at a suitable distance and strength between the RF gun and the acceleration section [75].

For Gaussian-shaped bunches, the expression for the radial force (64) is quite different. The space-charge-induced force is now highly nonlinear; a simple compensation of the emittance growth is not possible. Effort has been made to shape the cathode laser beam in the transverse and the longitudinal dimension to obtain a cylindrical electron distribution.

A detailed analysis shows that the emittance growth is due to a misalignment of the phase space ellipses of longitudinal slices of the electron bunch [76]. The effect of the solenoid is to counteract this misalignment.

A measurement of the relative rotation of the slice ellipses leading to emittance compensation has been performed at BNL [77]. The experiment showed that the phase space ellipses of longitudinal bunch slices indeed rotate relative to each other as the solenoid field is changed, and that they go through near-alignment at a particular solenoid field.

This so-called emittance compensation technique has been analytically described using envelope equations [78]. A particular solution is called the "invariant envelope," which can be used to optimize the design of RF-gun-based photoinjectors.

An interesting result of the theoretical description is that in the space-charge-dominated regime mismatches between the space-charge-correlated forces and the external RF focusing gradient produce 
slice envelope oscillations. This results in oscillations of the normalized emittance. In order to damp the emittance oscillations, the beam has to be injected into the accelerator or booster with a laminar envelope waist. At the same time, the accelerating field $E_{\text {acc }}$ has to be properly matched to the beam size, energy, and peak current. This is described by the matching condition

$$
E_{\mathrm{acc}}=\frac{1}{\sigma_{\mathrm{b}}} \sqrt{\frac{I_{0}}{2 I_{\mathrm{A}} \gamma}} .
$$

The emittance oscillation has been experimentally verified at the SPARC facility [79].

With these techniques in hand, several facilities built RF-gun-based injectors and achieved normalized emittances below $1 \mu \mathrm{m}$ for bunch charges in the $0.1-1 \mathrm{nC}$ range.

One remarkable example is the LCLS RF gun [41]. The measured projected emittance for a bunch charge of $250 \mathrm{pC}$ is $\epsilon_{\mathrm{n}}=0.7 \mu \mathrm{m}$. The initial peak current is $I_{0}=30 \mathrm{~A}$, with a bunch length of $0.75 \mathrm{~mm}$ (rms). At the photoinjector test stand PITZ at DESY (Zeuthen site), a projected emittance of $\epsilon_{\mathrm{n}}=0.9 \mu \mathrm{m}$ has been measured for a charge of $1 \mathrm{nC}$ [40], meeting the requirements for the European XFEL.

As discussed above, a small projected emittance is usually obtained with cylindrical electron beam shapes. Even with the emittance compensation scheme applied, the emittance of a Gaussianshaped electron bunch is larger at the tails of the Gaussian distribution. This is illustrated in Fig. 16:

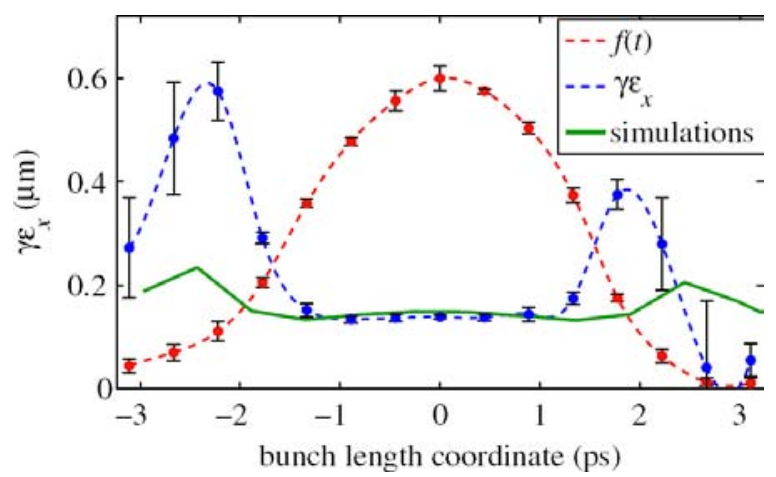

Fig. 16. Example of a slice emittance measurement at LCLS. Shown are the measured (blue dots) and the simulated (green curve) time-sliced horizontal emittance along the length of the bunch. The charge is $20 \mathrm{pC}$ and the UV laser spot diameter on the cathode is $0.6 \mathrm{~mm} ; f(t)$ is the bunch temporal distribution on an arbitrary scale. (Reprinted from Refs. 41 and 100.) for a Gaussian-shaped longitudinal charge distribution, the emittance at the head and tail is larger by a factor of 2-3 than in the center, leading to a large projected emittance.

In practice, a small emittance of the center slice may be sufficient to fulfill the emittance criterion (51) - if this slice has at the same time a high peak current and a small energy spread.

It is important to note that the projected emittance measured at the gun exit or after the booster - but before compression - is nevertheless a good estimate of the relevant emittance of the part or slice of the bunch which actually lases. After compression, due to space charge or coherent radiation effects, the projected emittance of the whole bunch may be spoiled. Nevertheless, it is possible to arrange the compression in such a way that the compressed part with high peak current keeps its small slice emittance and thus is able to produce SASE radiation.

A nice time-resolved measurement of the electron phase space after compression at FLASH [85] has shown that indeed the core emittance of the lasing high current peak is almost as small as the initial projected emittance.

\section{Bunch Compression and Beam Dynamics Aspects}

As discussed in the previous section, the peak current at the electron source with a beam energy of $5 \mathrm{MeV}$ is usually below $100 \mathrm{~A}$. The bunch charge is in the range of $0.1-1 \mathrm{nC}$, and the rms bunch length $\sigma_{z}=1 \ldots 2 \mathrm{~mm}$. According to Eq. (64), space charge forces scale with $1 / \sigma_{z} \gamma^{2}$, which implies compressing the bunches to the required $\mathrm{kA}$ level after acceleration at high energy.

\subsection{Compression}

At high energies, compression is obtained by magnetic chicanes. Off-crest acceleration induces an energy chirp along the bunch such that the trailing particles move on a shorter trajectory through the chicane than the leading particles.

A linear energy chirp is defined as

$$
\xi=\frac{d \eta}{d z},
$$

with the energy deviation $\eta$ [Eq. (12)]; $z$ is, as usual, the coordinate along the bunch. In a magnetic 
chicane with a momentum compaction factor $R_{56}$ the position of the particles in the bunch after the chicane $z_{f}$ is then

$$
z_{f}=z_{i}+R_{56} \eta=z_{i}\left(1+\xi R_{56}\right)+R_{56} \eta_{i},
$$

where $z_{i}$ is the initial position in the bunch, and the additional term takes into account the initial uncorrelated energy spread $\eta_{i}$. From this we can calculate the final rms bunch length $\sigma_{f}$ (see for instance [80]):

$$
\sigma_{f}=\sqrt{\left(1+\xi R_{56}\right)^{2} \sigma_{i}^{2}+R_{56}^{2} \sigma_{\eta, i}^{2}}
$$

where $\sigma_{i}$ is the initial rms bunch length and $\sigma_{\eta, i}$ the uncorrelated rms relative intrinsic energy spread of the bunch.

For optimal compression, the final bunch length is limited by the initial uncorrelated energy spread.

Neglecting the initial energy spread, the compression factor $C$ is then given by

$$
C=\frac{1}{1+\xi R_{56}} .
$$

Due to the applied energy chirp, the correlated energy spread of the bunch increases with compression.

However, because of the cosine-like RF wave, offcrest acceleration induces a nonlinear cosine-shaped energy chirp. This is corrected by RF cavities operating with a third harmonic of the fundamental RF wavelength.

In practice, the bunch is not fully compressed in a single chicane. A single full compression at high energy would lead to an undesired large energy spread, and compression at low energy to large space charge forces.

Therefore, usually two chicanes at different beam energies are used. As an example for a compression scheme, we take LCLS parameters from [86]. The bunch from the injector with a charge of $250 \mathrm{pC}$ and a length of $\sigma_{i}=750 \mu \mathrm{m}$ is compressed by two magnetic chicane compressors at a beam energy of $250 \mathrm{MeV}$ and $4.5 \mathrm{GeV}$, first to $\sigma_{f}=100 \mu \mathrm{m}$ and finally to $8 \mu \mathrm{m}$. The momentum compaction of the two chicanes is $R_{56}=-45.5 \mathrm{~mm}$ and $R_{56}=-24.7 \mathrm{~mm}$, respectively. The peak current increases from $43 \mathrm{~A}$ to $3.7 \mathrm{kA}$ with a compression factor $C_{1}=7$ and $C_{2}=12.5$. The energy spread increases to $3.5 \mathrm{MeV}$ and $16 \mathrm{MeV}$, respectively.

\subsection{Coherent synchrotron radiation}

Although the compression scheme is simple and efficient, collective effects of relativistic electron bunches have to be taken into account.

As discussed in Subsec. 3.1, coherent synchrotron radiation (CSR) is emitted in a dipole magnet for wavelengths longer than the electron bunch length. A Gaussian bunch induces an average energy loss per electron per unit length of [86]

$$
\frac{d W}{d s} \approx \frac{1.8}{4 \pi \epsilon_{\mathrm{o}}} \frac{N_{\mathrm{e}} e^{2}}{r^{2 / 3} \sigma_{z}^{4 / 3}},
$$

where $r$ is the dipole bending radius and $s$ the coordinate along the curved particle trajectory. Taking LCLS parameters of the first compressor, this amounts to a considerable loss of $2 \mathrm{MeV}$ at the last dipole if the bunch is compressed to $10 \mu \mathrm{m}$.

Coherent radiation is also emitted at short wavelength if the electron beam has highfrequency density modulations caused for instance by microbunching instabilities [88]. A high-brightness electron beam with a small amount of longitudinal density modulation can create self-fields that lead to energy modulations. This can be induced by collective effects like longitudinal space charge, CSR, or geometrical wakefields. Saldin et al. made the conclusion that the effect of longitudinal space charge on the microbunching instability is stronger than the effects of CSR and of geometrical wakefields for typical FEL linac and bunch compressor parameters [89].

In a magnetic chicane the energy modulation is converted into a density modulation, which can be much larger than the initial modulation. This may lead to many unwanted effects, like the breakup of bunches, or an increase in energy spread and emittance.

The amplification of microbunch instabilities is a serious concern, since the performance of a high-gain FEL crucially depends on the beam properties.

Detailed measurements of CSR-induced energy loss and transverse emittance growth at the two LCLS bunch compressors have been presented by Bane et al. [86]. An overview of CSR simulation codes can be found in [87].

An important finding is that the microbunching instability is very sensitive to a small uncorrelated energy spread of the electron beam. The uncorrelated 


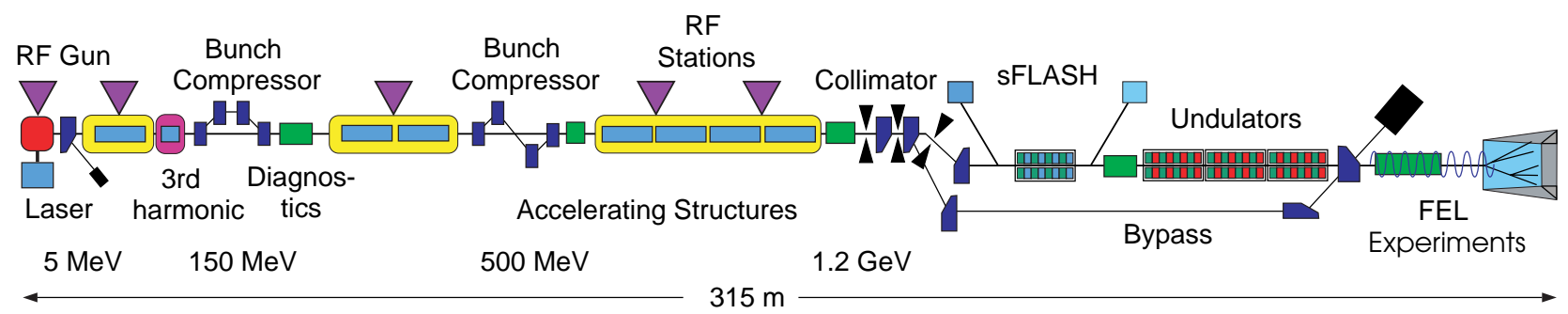

Fig. 17. Schematic layout of FLASH (not to scale); the electron beam direction is from left to right. The total length of the facility, including the experimental hall, is $315 \mathrm{~m}$.

energy spread from an RF-gun-based injector is very small, typically about $3 \mathrm{keV}$. In order to fully suppress the instabilities, Saldin et al. proposed increasing the uncorrelated energy spread to about $20 \mathrm{keV}$ applying a strong damping with resonant laserelectron interaction in an undulator, the so-called laser heater [89].

Such a laser heater has been realized at LCLS [90], which confirmed that a laser heater adequately damps the microbunching instability, resulting in more FEL power when switched on [38].

To mitigate the CSR effects, a compression scheme using velocity bunching has been proposed [81], and experimentally tested [82]. It could also be shown that by proper matching the expected emittance growth can be kept small [83].

The SCSS prototype FEL facility uses a thermionic pulsed $500 \mathrm{kV}$ electron gun with a heated cathode together with an appropriate bunching scheme [42]. Microbunch instabilities have not been observed at SCSS [84].

\section{The Soft X-ray Facility FLASH}

As discussed in the introduction, many facilities for soft and hard X-rays are under construction or in the planning phase.

As an example, the user facility FLASH at DESY, Germany, will be discussed. To a certain extent, FLASH can serve as a blueprint for many facilities. Like all FELs, FLASH can be divided into five basic sections: the electron source, the linac to accelerate the electron bunches, a bunch compressor to provide high peak currents, the undulator system to produce the FEL radiation, and one or more endstations to use the radiation for research purposes. The layout of FLASH is shown in Fig. 17. Table 1
Table 1. FLASH parameters.

\begin{tabular}{lll}
\hline Electron beam & & \\
Energy & $\mathrm{MeV}$ & $370-1200$ \\
Peak current & $\mathrm{kA}$ & 2.5 \\
Emittance (rms), norm & $\mu \mathrm{m}$ & 1.4 \\
Energy spread (rms) & $\mathrm{keV}$ & $<200$ \\
Bunch train length & $\mu \mathrm{s}$ & $1-800$ \\
Number of bunches per train & & $1-800(2400)$ \\
Repetition rate & $\mathrm{Hz}$ & 10 \\
Undulator & & \\
Period & $\mathrm{cm}$ & 2.73 \\
Gap & $\mathrm{mm}$ & 12 \\
Peak magnetic field & $\mathrm{T}$ & 0.48 \\
K & & 1.23 \\
Total magnet length & $\mathrm{m}$ & 27 \\
FEL radiation delivered to experiments (2nd user run $)$ \\
Wavelength (fundamental) & $\mathrm{nm}$ & $47-6.8$ \\
Smallest harmonics & $\mathrm{nm}$ & 1.59 \\
Average pulse energy & $\mu \mathrm{J}$ & $10-100$ \\
Bandwidth (FWHM) & $\%$ & 1 \\
Pulse duration (FWHM) & fs & $10-50$ \\
Peak power & $\mathrm{GW}$ & $1-5$ \\
Peak spectral brilliance & $*$ & $\sim 10^{29}-10^{30}$ \\
& &
\end{tabular}

*Photons $/ \mathrm{s} \mathrm{mrad}^{2} \mathrm{~mm}^{2}(0.1 \% \mathrm{bw})$.

summarizes the main parameters of FLASH. For completeness, Table 2 shows measured parameters of LCLS and design parameters for the European XFEL and the SPring-8 XFEL.

FLASH is a burst mode machine. It uses superconducting accelerating technology. This allows the acceleration of bursts of several hundred bunches within a 0.8 -ms-long pulse train, 10 trains per second. For a more complete description of FLASH, see $[32,34,91]$ and references therein.

\subsection{Electron source}

The electron source is a laser-driven RF gun photoinjector. A 1.5-cell normal conducting $1.3 \mathrm{GHz} \mathrm{L}$-band 
Table 2. Parameters of hard X-ray FEL facilities. LCLS is in operation, while the European XFEL and the SPring-8 XFEL are under construction.

\begin{tabular}{|c|c|c|c|c|}
\hline Facility & & $\begin{array}{c}\text { LCLS- } \\
\text { measured }[38,39]\end{array}$ & $\begin{array}{c}\text { European XFEL } \\
\text { design [14] }\end{array}$ & $\begin{array}{c}\text { SPring-8 XFEL } \\
\text { design [92] }\end{array}$ \\
\hline \multicolumn{5}{|l|}{ Electron beam } \\
\hline Energy & $\mathrm{GeV}$ & 13.6 & 17.5 & 8 \\
\hline Peak current & $\mathrm{kA}$ & $2.5-3.5$ & 5 & 3 \\
\hline Emittance (rms, norm, inj.) & $\mu \mathrm{m}$ & $0.4^{\dagger}$ & 0.9 & 0.8 \\
\hline Energy spread (rms) & $\%$ & 0.04 & 0.011 & \\
\hline Bunch train length & $\mu \mathrm{s}$ & - & 600 & 0.2 \\
\hline Number of bunches per train & & 1 & 2700 & $1(50)$ \\
\hline Repetition rate & $\mathrm{Hz}$ & $30(120)$ & 10 & 60 \\
\hline \multicolumn{5}{|l|}{ Undulator } \\
\hline Type & & Planar & Planar (SASE 1) & Planar \\
\hline Period & $\mathrm{cm}$ & 3.0 & 3.56 & 1.8 \\
\hline Gap & $\mathrm{mm}$ & 6.8 & 10 & $4(2-40)$ \\
\hline Peak magnetic field & $\mathrm{T}$ & 1.25 & 1.0 & 1.13 \\
\hline$K$ & & 3.5 & 3.32 & 1.9 \\
\hline Total undulator length & $\mathrm{m}$ & 112 & 200 & $90(120)$ \\
\hline \multicolumn{5}{|l|}{$F E L$ radiation } \\
\hline Wavelength (fundamental) & $\mathrm{nm}$ & 0.15 & 0.1 & 0.1 \\
\hline Photon pulse energy & $\mathrm{mJ}$ & $1.5-3.0$ & 1.7 & 0.4 \\
\hline Bandwidth (FWHM) & $\%$ & $0.2-0.5$ & 0.08 & \\
\hline Pulse duration (rms) & fs & $30-40$ & 40 & $20-200$ \\
\hline Number of photons per pulse & & $2 \cdot 10^{12}$ & $0.8 \cdot 10^{12}$ & $2 \cdot 10^{11}$ \\
\hline Peak power & GW & $15-40$ & 15.6 & 20 \\
\hline Peak spectral brilliance & $*$ & $2 \cdot 10^{33}$ & $2.1 \cdot 10^{33}$ & $1 \cdot 10^{33}$ \\
\hline
\end{tabular}

${ }^{*}$ Brightness is photons $/ \mathrm{s} \mathrm{mrad}^{2} \mathrm{~mm}^{2}(0.1 \%$ bandwidth $)$.

${ }^{\dagger}$ Refers to femtosecond-scale time slices.

copper cavity is powered by a $5 \mathrm{MW}$ klystron, pulsed with $10 \mathrm{~Hz}$ with a pulse duration of up to $850 \mu$ s. The photocathode is a thin film of $\mathrm{Cs}_{2} \mathrm{Te}$ deposited on a molybdenum plug, inserted in the RF gun backplane via a load-lock system. The laser is based on a mode-locked pulse train oscillator synchronized to the $1.3 \mathrm{GHz} \mathrm{RF}$ of the accelerator. A chain of diodepumped Nd:YLF amplifiers provides enough power to convert the initial infrared wavelength into ultraviolet $(262 \mathrm{~nm})$.

The electron bunch charge is variable to a certain extent: a charge between $0.2 \mathrm{nC}$ and $1 \mathrm{nC}$ is used during FEL operation. The number of electron bunches per bunch train can be varied from a single bunch up to 800 , in a special mode 2400 . Since the bunch train length is limited to $800 \mu \mathrm{s}$, the maximum number of bunches in a train depends on the spacing between the bunches: several distinct spacings corresponding to frequencies between $3 \mathrm{MHz}$ and $40 \mathrm{kHz}$ are possible. The projected normalized transverse rms emittance for a $1 \mathrm{nC}$ bunch is $1.4 \mu \mathrm{m}$.

\subsection{Acceleration}

FLASH uses TESLA-type superconducting accelerating modules. Each module consists of eight nine-cell standing wave niobium cavities with a fundamental mode frequency of $1.3 \mathrm{GHz}$. Each 1-m-long cavity is equipped with an RF power coupler, a pickup probe, two high-order mode couplers, and a motorized tuning system. Many cavities are equipped with piezoelectric tuners as well. The cavities are bath-cooled by superfluid helium to $2 \mathrm{~K}$. The length of a complete accelerating module is $12 \mathrm{~m}$, including a quadrupole doublet, two dipole correctors, and a beam position monitor.

The electron beam energy of $5 \mathrm{MeV}$ from the $\mathrm{RF}$ gun is boosted to $150 \mathrm{MeV}$ by the first accelerating module.

The first module is followed by four thirdharmonic cavities operated at $3.9 \mathrm{GHz}$. These are used to linearize the energy chirp required for bunch compression. A voltage of up to $21 \mathrm{MV}$ can be 


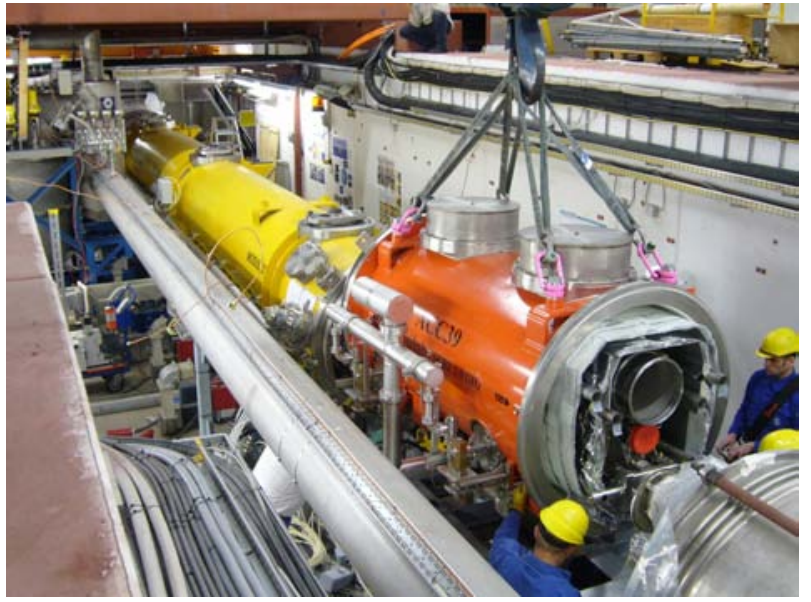

Fig. 18. Installation of the cryomodule containing four $3.9 \mathrm{GHz}$ superconducting cavities into the FLASH injector in 2009. (Courtesy of Kay Jensch, DESY.)

applied. Figure 18 shows the installation of the $3.9 \mathrm{GHz}$ module into the FLASH injector in 2009 .

The next two accelerator modules are located between the bunch compressors increasing the electron beam energy to $500 \mathrm{MeV}$. The second bunch compressor is followed by a string of four modules to accelerate the electrons up to $1.2 \mathrm{GeV}$. The modules show excellent performance with an average gradient of $25 \mathrm{MV} / \mathrm{m}$. Several cavities reach gradients exceeding $30 \mathrm{MV} / \mathrm{m}$, approaching the limit for this type of superconducting cavities.

The RF is provided by five RF stations. Each of them has a $5 \mathrm{MW}$ klystron (one station is equipped with a multibeam 10 MW klystron), a high-voltage pulse transformer, and a bouncer-type pulsed power supply (modulator). The RF pulse duration is $1.5 \mathrm{~ms}$. The loaded quality factor of the superconducting cavities is adjusted to $3 \cdot 10^{6}$, leading to a filling time of $500 \mu \mathrm{s}$; the flat top part for acceleration is $800 \mu \mathrm{s}$.

FLASH uses superconducting accelerator modules and RF systems similar to those that will be used for the European XFEL and the International Linear Collider, providing thus an important test bench for these facilities.

A dedicated low-level RF system stabilizes and flattens with feedback and feedforward methods the amplitude and phase of the accelerating field in the RF gun, the third-harmonic cavities, and the accelerator modules.

The regulation and stabilization at the $\mathrm{RF}$ gun and of the first module are especially important. For the RF gun, an rms phase stability of $0.02^{\circ}$ and an amplitude stability of better than $0.1 \%$ are achieved.

For those cavities in the modules which are driven by one klystron, the vector sum of amplitude and phase is computed. An excellent rms energy stability of $\sigma_{W} / W=1 \cdot 10^{-4}$ is achieved; the phase stability is $0.01^{\circ}$.

Because of the bunch compressor chicane with an $R_{56}$ of $0.18 \mathrm{~m}$, the energy jitter is translated into an arrival time rms jitter of $60 \mathrm{fs}$. An optical synchronization system based on the bunch arrival time detection is being set up at FLASH, with the goal of stabilizing the arrival time of all bunches in the pulse train to the $10 \mathrm{fs}$ level. This is important for proper acceleration of long pulse trains, for seeding schemes with external lasers, and for pump-probe experiments.

\subsection{Bunch compression}

Two magnetic chicane bunch compressors are used. The first compression is at $150 \mathrm{MeV}$, the second at $500 \mathrm{MeV}$.

Different compression schemes are applied. Before the installation of the third-harmonic cavities in early 2010, rollover compression at bunch charges of $0.5-1 \mathrm{nC}$ was used. Because of the cosine shape of the energy chirp, a sharp spike with a long tail develops. Only a small fraction of the charge is contained in the spike. This spike is very short. The FEL pulse length generated by this spike has been measured to be $30 \mathrm{fs}$; in some cases the estimated pulse duration was as short as $5 \mathrm{fs}$.

The new third-harmonic cavities to flatten the energy chirp allow more flexible adjustment of the compression. To achieve different bunch lengths, the bunch charge and the compression factor are varied. For smaller charges, a stronger compression can be applied without spoiling the bunch due to space charge effects. At $1 \mathrm{nC}$, the rms FEL pulse duration is expected to be $30 \mathrm{fs}$; for $0.2 \mathrm{nC}$ it is $7 \mathrm{fs}$. The energy in the FEL pulse is expected to be about $1 \mathrm{~mJ} / \mathrm{nC}$, which is in good agreement with recent measurements at FLASH.

FLASH can produce bunches with smaller charges; $20 \mathrm{pC}$ are discussed to approach $1 \mathrm{fs}$ short FEL pulses. Unfortunately, most electron beam instrumentation devices at FLASH (BPMs, OTR screens) cannot yet measure bunches with such a low charge. An upgrade of instrumentation and 
diagnostics, and beam tests with very small bunch charges, are planned.

\subsection{Undulators}

Six fixed-gap undulator modules, each with a length of $4.5 \mathrm{~m}$, are installed. The undulator consists of a periodic structure of permanent $\mathrm{NdFeB}$ magnets with a gap of $12 \mathrm{~mm}$. The peak magnetic field is $0.47 \mathrm{~T}$, the undulator period $27.3 \mathrm{~mm}$, and the $K$ value 1.23. An excellent field quality has been achieved; the deviation from the ideal orbit is below $10 \mu \mathrm{m}$. The field is almost purely sinusoidal, and the contributions from odd harmonics are very small, below $0.1 \%$ (third) and below $0.05 \%$ (fifth).

Between the undulators a diagnostic block is installed with quadrupoles for beam focusing.

With a beam energy between $370 \mathrm{MeV}$ and $1.2 \mathrm{GeV}$, a wavelength between $47 \mathrm{~nm}$ and $4.45 \mathrm{~nm}$ is achieved. The third, and sometimes the fifth harmonic of the fundamental wavelength, are also used for experiments.

\subsection{Photon diagnostics}

The undulator is followed by a photon diagnostics section and a photon beamline to transport the FEL radiation to the experimental hall, where the user experiments are located. The transverse size and position of the photon beam are measured at several locations using Ce:YAG screen monitors. The energy of the FEL radiation pulses is measured either by a detector based on gold wires and a microchannel plate (MCP) or by an absolutely calibrated gasmonitor detector. It is based on photoionization of gases with well-known cross-sections. Both detectors have a large dynamic range, covering several orders of magnitude from spontaneous emission to saturation. The FEL radiation spectrum is measured by a high-resolution spectrometer. Online, nondestructive spectrometers are also available.

The distance to the experiments is large enough to allow safe use of grazing incidence mirrors. The beam can be directed into one of the five experimental beam lines.

The facility is hosting many experiments, ranging from atomic physics through materials science to biology. Since 2005, 14,000 h of beam time have been delivered to users. In the second user period, from 2007 to 2009, the facility achieved an overall uptime of $93 \%$. More than 30 different wavelengths have been set up; at the request of the users, the wavelength has been changed more than 140 times, and many different bunch patterns within the bunch train have been realized.

\subsection{Outlook}

The third user period of FLASH has started in September 2010 and will provide more than $4000 \mathrm{~h}$ of beam time for user experiments. In late 2011, the construction of FLASH II, the second-undulator beam line, will begin. It will make full use of the existing accelerator of FLASH. Parts of the bunch trains are separated from the main beam line into the new undulator beam line with a shallow angle. The main feature of FLASH II will be the production of seeded FEL radiation and, in a later stage, circular polarization. All undulators will have a variable gap, in order to obtain reasonable flexibility in the choice and tuning of the wavelength. A separate experimental hall is planned for an additional set of experimental stations making use of the new undulator line [94].

\section{Acknowledgments}

I would like to thank the numerous colleagues around the world working on free electron lasers, contributing to the wonderful success of X-ray lasers. I am especially grateful to Peter Schmüser and Katja Honkavaara for their support, proofreading, and fruitful discussions, and Romain Schreiber for helping with data plotting.

\section{References}

[1] J. M. J. Madey, J. Appl. Phys. 42, 1906 (1971).

[2] L. R. Elias, W. M. Fairbank, J. M. J. Madey, H. A. Schwettman and T. I. Smith, Phys. Rev. Lett. 36, 717 (1976).

[3] D. A. G. Deacon et al., Phys. Rev. Lett. 38, 892 (1977).

[4] A. M. Kondratenko and E. L. Saldin, Part. Accel. 10, 207 (1980).

[5] Y. S. Derbenev, A. M. Kondratenko and E. L. Saldin, Nucl. Instrum. Methods 193, 415 (1982).

[6] R. Bonifacio, C. Pellegrini and L. M. Narducci, Opt. Commun. 50, 373 (1984).

[7] J. B. Murphy and C. Pellegrini, Nucl. Instrum. Methods A 237, 159 (1985).

[8] J. B. Murphy and C. Pellegrini, J. Opt. Soc. Am. B 2, 259 (1985). 
[9] K.-J. Kim, Phys. Rev. Lett. 57, 1871 (1986).

[10] K.-J. Kim, Nucl. Instrum. Methods A 250, 396 (1986).

[11] C. Pellegrini, Nucl. Instrum. Methods A 272, 364 (1988).

[12] LCLS website: https://slacportal.slac.stanford. edu/sites/lcls_public/Pages/Default.aspx

[13] SPring-8 XFEL website, http://www-xfel.spring8. or.jp; SCSS Conceptual Design Report, http:// www-xfel.spring8.or.jp/SCSSCDR.pdf

[14] European XFEL website: http://www.xfel.eu

[15] FLASH website: http://flash.desy.de

[16] Fermi at Elettra website, http://www.elettra. trieste.it / FERMI / index.php?n= Main.HomePage; Fermi at Elettra, Conceptual Design Report, ST/F-TN-07/12; http://www.elettra.trieste.it/ fermi_old/index.php?n=Main.CDRdocument

[17] Swiss FEL website: http://www.psi.ch/media/ swissfel-the-future-project

[18] New Light Source website, http://www. newlightsource.org; NLC conceptual design report, http://www.newlightsource.org/documents/NLS \%20CDR\%20Consolidated\%20Final.pdf

[19] A list of free electron lasers can be found at http:// sbfel3.ucsb.edu/www/vl_fel.html; other useful information on light sources may be obtained at http://www.lightsources.org

[20] T. J. Orzechowski et al., Phys. Rev. Lett. 54, 889 (1985).

[21] M. Hogan et al., Phys. Rev. Lett. 80, 289 (1998).

[22] D. C. Nguyen et al., Phys. Rev. Lett. 81, 810 (1998).

[23] M. Hogan et al., Phys. Rev. Lett. 81, 4867 (1998).

[24] S. V. Milton et al., Science 292, 2037 (2001).

[25] A. H. Lumpkin et al., Phys. Rev. Lett. 88, 234801 (2002).

[26] A. Tremaine et al., Phys. Rev. Lett. 88, 204801 (2002).

[27] L. DiMauro et al., Nucl. Instrum. Methods A 507, 15 (2003).

[28] J. Andruszkow et al., Phys. Rev. Lett. 85, 3825 (2000).

[29] V. Ayvazyan et al., Phys. Rev. Lett. 88, 104802 (2002).

[30] V. Ayvazyan et al., Eur. Phys. J. D 20, 149 (2002).

[31] V. Ayvazyan et al., Eur. Phys. J. D 37, 297 (2006).

[32] W. Ackermann et al., Nat. Photon. 1, 336 (2007).

[33] S. Schreiber, B. Faatz and K. Honkavaara, Operation of FLASH at $6.5 \mathrm{~nm}$ wavelength, in Proc. 11th European Particle Accelerator Conference (EPAC 08) (Genoa, Italy; 23-27 June 2008), MOPC030.

[34] K. Honkavaara et al., FLASH upgrade, in Proc. 1st Int. Particle Accelerator Conference (IPAC 10) (Kyoto, Japan; 23-28 May 2010), TUOARA01.

[35] For a list of publications on experiments conducted at FLASH, see http://hasylab.desy.de/facilities/ flash/publications/selected_publications.
[36] T. Shintake, First lasing at SCSS, in Proc. 28th Int. Free Electron Laser Conference (FEL 2006) (Berlin, Germany; 27 Aug.-1 Sep. 2006), MOAAU04.

[37] H. Tanaka et al., Operation status of the SCSS test accelerator: continuous saturation of SASE FEL at the wavelength range from 50 to 60 nanometers, in Proc. 11th European Particle Accelerator Conference (EPAC 08) (Magazzini del Cotone, Genoa, Italy; 23-27 June 2008), WEOAM01.

[38] P. Emma, First lasing of the LCLS X-ray FEL at $1.5 \mathrm{~A}$, in Proc. 23rd Particle Accelerator Conference ( $P A C$ 09) (Vancouver, Canada; 4-8 May 2009).

[39] P. Emma et al., Nat. Photon. (1 Aug. 2010).

[40] F. Stephan et al., Phys. Rev. ST Accel. Beams 13, 020704 (2010).

[41] Y. Ding et al., Phys. Rev. Lett. 102, 254801 (2009).

[42] T. Shintake et al., Phys. Rev. STAB 12, 070701 (2009).

[43] P. Schmüser, M. Dohlus and J. Rossbach, Ultraviolet and Soft X-Ray Free-Electron Lasers: Introduction to Physical Principles, Experimental Results, Technological Challenges, Springer Tracts in Modern Physics 229 (Springer, Berlin, Heidelberg, 2008) [DOI: 10.1007/978-3-540-79572-8].

[44] E. L. Saldin, E. A. Schneidmiller and M. V. Yurkov, The Physics of Free Electron Lasers (Springer, Berlin, Heidelberg, 2000).

[45] J. B. Murphy and C. Pellegrini, Introduction to the physics of the free electron laser, in Laser Handbook, eds. W. B. Colson, C. Pellegrini and A. Renieri, Vol. 6 (North-Holland, Amsterdam, Oxford, New York, Tokyo, 1990), p. 11ff.

[46] W. B. Colson, Classical free electron laser theory, in Laser Handbook, eds. W. B. Colson, C. Pellegrini and A. Renieri, Vol. 6 (North-Holland, Amsterdam, Oxford, New York, Tokyo, 1990), p. 115ff.

[47] Z. Huang and K.-L. Kim, Phys. Rev. ST Accel. Beams 10, 034801 (2007).

[48] W. A. Barletta, Nucl. Instrum. Methods A 618, 69 (2010).

[49] M. Trovò et al., Nucl. Instrum. Methods A 483 , 157 (2010).

[50] R. Colella and A. Luccio, Nucl. Instrum. Methods A 50, 41 (1984).

[51] K.-J. Kim, Y. Shvydko and S. Reiche, Phys. Rev. Lett. 100, 244802 (2008).

[52] W. Fawley, Rep. LBNL-49625 (Lawrence Berkeley Laboratory, 2002).

[53] S. Reiche, Nucl. Instrum. Methods A 429, 243 (1999).

[54] E. L. Saldin, E. A. Schneidmiller and M. V. Yurkov, Nucl. Instrum. Methods A 429, 233 (1999).

[55] S. Krinsky and Z. Huang, Phys. Rev. ST Accel. Beams 6, 050702 (2003). 
[56] E. L. Saldin, E. A. Schneidmiller and M. V. Yurkov, Phys. Rev. ST Accel. Beams 9, 050702 (2006).

[57] D. A. Edwards and M. J. Syphers, An Introduction to the Physics of High Energy Accelerators (John Wiley, New York, 1993).

[58] K. Floettmann, Phys. Rev. ST Accel. Beams 6, 034202 (2003).

[59] E. D. Courant and H. S. Snyder, Ann. Phys. 3, 1 (1958).

[60] Z. Huang and K.-J. Kim, Nucl. Instrum. Methods A 483, 504 (2002).

[61] M. Xie, Nucl. Instrum. Methods A 445, 59 (2000).

[62] E. L. Saldin, E. A. Schneidmiller and M. V. Yurkov, Opt. Commun. 235, 415 (2004).

[63] K.-J. Kim and M. Xie, Nucl. Instrum. Methods A 331, 359 (1993).

[64] R. Mitzner et al., Opt. Express 16, 19909 (2008).

[65] R. Mitzner et al., Phys. Rev. A 80, 025402 (2009).

[66] Ulrike Frühling et al., Nat. Photon. 3, 523 (2009).

[67] E. L. Saldin, E. A. Schneidmiller and M. V. Yurkov, Opt. Commun. 148, 383 (1998).

[68] A. Singer et al., Phys. Rev. Lett. 101, 254801 (2008).

[69] E. L. Saldin, E. A. Schneidmiller and M. V. Yurkov, Opt. Commun. 281, 1179 (2008).

[70] J. S. Fraser, R. L. Sheffield and E. R. Gray, Nucl. Instrum. Methods A 250, 71 (1986).

[71] E. Chevallay et al., Nucl. Instrum. Methods A 340, 146 (1994).

[72] D. Sertore et al., Nucl. Instrum. Methods A 445, 422 (2000).

[73] S. Lederer et al., Photocathode studies at FLASH, in Proc. 11th European Particle Accelerator Conference (EPAC 08) (Genoa, Italy; 23-27 June 2008), MOPC072.

[74] S. Schreiber et al., Nucl. Instrum. Methods A 445, 427 (2000).

[75] B. E. Carlsten, Nucl. Instrum. Methods A 285, 313 (1989).

[76] K.-J. Kim, Nucl. Instrum. Methods A 275, 201 (1989).

[77] X. Qiu, K. Batchelor, I. Ben-Zvi and X.-J. Wang, Phys. Rev. Lett. 76, 3723 (1996).

[78] L. Serafini and J. B. Rosenzweig, Phys. Rev. E 55, 7565 (1997).

[79] M. Ferrario et al., Phys. Rev. Lett. 99, 234801 (2007).

[80] B. Beutner, Measurement and analysis of coherent synchrotron radiation effects at FLASH. PhD thesis, University of Hamburg (2007); DESYTHESIS-2007-040 (Dec. 2007), http://www.phys net.uni-hamburg.de/services/fachinfo/__Kurzfass ungen/Bolko__Beutner.htm

[81] L. Serafini and M. Ferrario, AIP Conf. Proc. 581, 87 (2001).

[82] H. Iijima et al., Jpn. J. Appl. Phys. 44, 5249 (2005).

[83] M. Ferrario et al., Phys. Rev. Lett. 104, 054801 (2010).

[84] T. Shintake, private commun.

[85] M. Roehrs et al., Phys. Rev. ST Accel. Beams 12, 050704 (2009).

[86] K. L. F. Bane et al., Phys. Rev. ST Accel. Beams 12, 030704 (2009).

[87] G. Bassia et al., Nucl. Instrum. Methods A 557, 189 (2006).

[88] E. L. Saldin, E. A. Schneidmiller and M. V. Yurkov, Nucl. Instrum. Methods A 490, 1 (2002).

[89] E. L. Saldin, E. A. Schneidmiller and M. V. Yurkov, Nucl. Instrum. Methods A 528, 355 (2004).

[90] Z. Huang et al., Phys. Rev. ST Accel. Beams 7, 074401.

[91] K. Tiedtke et al., New J. Phys. 11, 023029 (2009).

[92] T. Shintake, Status report on Japanese XFEL construction project at SPring-8, in Proc. 1st Int. Particle Accelerator Conference (IPAC 10) (Kyoto, Japan; 23-28 May 2010), TUXRA02.

[93] http://www-ssrl.slac.stanford.edu/lcls/parameters. html

[94] B. Faatz et al., FLASH II: a seeded future at FLASH, in Proc. 1st Int. Particle Accelerator Conference (IPAC 10) (Kyoto, Japan; 23-28 May 2010), TUPE005.

[95] Adapted with kind permission of the European Physical Journal (EPJ), copyright EDP Sciences, Società Italiana di Fisica, Springer-Verlag 2005.

[96] Adapted by permission from Macmillan Publishers Ltd: Nature Photonics, [32], copyright (2007).

[97] Reprinted by permission from Macmillan Publishers Ltd: Nature Photonics, [39], copyright (2010).

[98] Reprinted with permission from [65]. Copyright (2009) by the American Physical Society.

[99] Reprinted with permission from [68]. Copyright (2008) by the American Physical Society.

[100] Reprinted with permission from [41]. Copyright (2009) by the American Physical Society. 
Siegfried Schreiber is a senior staff scientist at DESY, Hamburg, and since 2005 he has been responsible for the operation of the FEL FLASH. He has been active in the development and operation of RF-gun-based photoinjectors at CERN, FNAL, and DESY, mostly within the TESLA collaboration. He also participated in the machine-detector interface working group for the TESLA proposal. He made important contributions to upgrading the TTF1 VUV FEL to the user facility FLASH, and is currently working on further improvements and upgrades of the FLASH facility. Dr. Schreiber is a member of the ICFA Panel on Advanced and Novel Accelerators. 